Aus der Abteilung Allgemeinmedizin

(Prof. Dr. med. M.M. Kochen, MPH, FRCGP)

im Zentrum Innere Medizin

der Medizinischen Fakultät der Universität Göttingen

\title{
Patientenaufklärung und Stellenwert hausärztlicher Pharmakotherapien in kommunalen Krankenhäusern
}

INAUGURAL - DISSERTATION

zur Erlangung des Doktorgrades

der Medizinischen Fakultät

der Georg-August-Universität zu Göttingen

\author{
vorgelegt von \\ Uwe Sorns \\ aus \\ Mühlhausen
}

Göttingen 2001 
Dekan: Prof. Dr. med. M. Droese

I. Berichterstatter/in: Prof. Dr. med. M.M. Kochen, MHP, FRCGP

II. Berichterstatter/in: Prof. Dr. med. J. Brockmöller

Tag der mündlichen Prüfung: 25. April 2002 
Inhaltsverzeichnis

Seite

1. Einleitung

2. Stand der Forschung

2.1 Verordnungsverhalten von Krankenhausärzten

2.2 Verordnungsverhalten von Hausärzten

2.3 Probleme der Patienten

3. Fragestellung der Untersuchung

4. Material und Methoden

4.1 Krankenhäuser

4.2 Patienten

4.3 Datenerhebung

4.4 Patientenbefragung 
5. Ergebnisse

5.1 Patienten

5.2 Vorerkrankungen, Einweisungs- und Krankenhausdiagnosen 
Tabelle 3: Diagnosen und deren Häufigkeit im Vergleich zwischen Vorerkrankungen, Einweisungs- und Krankenhausdiagnosen

Tabelle 5: Arzneimittelverordnung und deren Häufigkeit in der Praxis

Tabelle 6: Veränderungen der Einweisungsmedikation im Krankenhaus

Tabelle 7: Veränderungen innerhalb der Medikamentengruppen

Tabelle 8: Abgesetzte Medikamente

Tabelle 9: Umstellung der Einweisungsmedikation im Krankenhaus auf Originalpräparate oder Generika

Tabelle 10: Arzneimittel- Neuverordnungen

Tabelle 11: Originalpräparate und Generika der Krankenhäuser (Neuverschreibungen) und der Hausarztpraxen

Tabelle 12: Aufklärung der Patienten über medikamentöse 
Tabelle 13: Bedeutung der Aufklärung für die Altersgruppen

Tabelle 14: Inhalt der Aufklärungsgespräche

Tabelle 15: Wissen der Patienten über Neuverordnungen

Tabelle 16: Wissen der Altersgruppen über Neuverordnungen 


\section{Einleitung}

Die Aufrechterhaltung oder Wiederherstellung der Gesundheit und somit auch des Wohlbefindens sind oberstes Ziel ärztlichen Handelns. Die medikamentöse Behandlung ist für dieses Ziel eine wichtige Therapieoption. Allgemeinärzte, die ca. $50 \%$ aller ambulant verordneten Medikamente verschreiben, sehen sich mit der Kritik konfrontiert, Medikamente mit z. T. ungesicherter Wirksamkeit sowie zu teure Arzneimittel zu verschreiben (Kochen 1998). Krankenhausärzte hingegen überschauen manchmal nicht die auf Hausärzte zukommenden Kosten einer Dauerbehandlung mit einer teuren, im Krankenhaus begonnenen Arzneitherapie (Jones R und Rawlins 1992). Die Einweisung eines hausärztlich betreuten Patienten in ein Krankenhaus dürfte nicht selten Konflikte dieser Art hervorrufen.

In vorangegangenen Arbeiten wurde auf dem Gebiet der Allgemeinmedizin die Thematik der hausärztlichen Medikation im Krankenhaus mehrfach aufgegriffen. Himmel et al. (1996b) stellten z.B. das "Schicksal hausärztlicher Verordnungen im Krankenhaus" aus Perspektive einer Allgemeinpraxis dar. In einer weiteren Studie von Himmel et al. (1996a) wurde die quantitative Bedeutung von Medikationsänderungen anhand von Sonderanforderungen in einem Universitätsklinikum aufgezeigt.

Die vorliegende Arbeit untersucht den Stellenwert hausärztlicher Pharmakotherapien in kommunalen Krankenhäusern. Die Medikation allgemeinärztlich eingewiesener Patienten vor und während ihres Krankenhausaufenthaltes wird verglichen.

Die Veränderung der hausärztlichen Pharmakotherapie nach Krankenhauseinweisung kann nicht nur das Verhältnis zwischen stationär und ambulant tätigen Ärzten belasten. Vor allem der Patient wird in dieser Situation häufig alleingelassen. Wenn Medikamente ohne nachvollziehbare Erklärung verändert werden, könnte dies den Patienten als Qualitätsminderung der Therapie erscheinen (Himmel und Kochen 1998). 
Doch ist gerade ein mit der ärztlichen Behandlung zufriedener Patient besser in der Lage, aktiv am Heilungsprozeß mitzuwirken (Hasenbring u. Ahrens 1986).

Was weiß der Patient über die Medikamente, die im Krankenhaus neu verschrieben werden, und in wieweit wurde er über Änderungen seiner Langzeitmedikation aufgeklärt? Diese Fragen zur Qualität der Patienteninformation sind der zweite Untersuchungsschwerpunkt dieser Studie.

\section{Stand der Forschung}

\subsection{Verordnungsverhalten von Krankenhausärzten}

Die medikamentöse Therapie von Klinikärzten unterscheidet sich häufig vom Verordnungsverhalten hausärztlicher Kollegen. Nach stationärer Einweisung kommt es oft zu Änderungen lang bestehender Behandlungsregimes: Medikamente werden abgesetzt oder durch andere Präparate ersetzt, Dosierungen geändert und neue Medikamente verordnet.

Vorliegende Studien zum Umfang und den Gründen der von Klinikärzten geänderten Medikation widersprechen sich teilweise. Besonders auffällig wird diese Widersprüchlichkeit, wenn die Größenordnung der Medikamentenänderung während eines Krankenhausaufenthaltes geschätzt wird. So untersuchten Beers et al. (1989) in einer amerikanischen Klinik die Veränderungen der Dauermedikation bei 197 ältereren Patienten zum Zeitpunkt der Einweisung sowie bei Entlassung. Während des Krankenhausaufenthaltes wurden $40 \%$ der vom Hausarzt verordneten Pharmaka abgesetzt. Die Klinikärzte begründeten dies unter anderem mit der Unwirksamkeit bzw. Toxizität einiger Medikamente. Signifikant waren die Änderungen in einzelnen Arzneimittelgruppen. So stiegen die Verschreibungen von Benzodiazepinen, Analgetika, Abführmitteln und Kardiaka zum Teil stark an. Die Zahl der verordneten Medikamente änderte sich insgesamt nur geringfügig: von durchschnittlich 4,5 bei Aufnahme auf 4,8 Präparate bei Entlassung. 
Dagegen ermittelten Gosney und Tallis (1984) in einer ähnlich angelegten englischen Studie, daß sich die Zahl der Medikamente deutlich erhöhte: von durchschnittlich 2,14 vor Einweisung auf 5,48 während des stationären Aufenthaltes. Am häufigsten verschrieben die Klinikärzten den Patienten Antibiotika $(70,1 \%)$, Analgetika $(65,4 \%)$ und Diuretika $(50,6 \%)$.

Im Vergleich zur ambulanten Behandlung registrierten auch Lucena Gonzalez et al. (1995) bei einer Untersuchung von 504 Patienten einer internistischen Abteilung in Spanien nahezu eine Verdopplung der verordneten Medikamente von durchschnittlich 3,3 auf 6 je Patient. Diese Zunahme betraf alle Arzneimittelgruppen, besonders jedoch Antibiotika, durchblutungs- und verdauungsfördernde Medikamente. Ein Zusammenhang des Wechsels von Medikamenten mit Alter und Geschlecht der Patienten konnte nicht entdeckt werden.

In ihrer Studie kommen Kruse et al. (1990) zu einem gegenteiligen Ergebnis. Demnach sank die Anzahl der Medikamente nach stationärer Aufnahme in eine geriatrische Heidelberger Klinik um ca. ein Drittel. Die Ärzte im Krankenhaus reduzierten hauptsächlich Antihypotonika, Antihypertonika, Digitalis, Expektoranzien, orale Antidiabetika, Diuretika und Benzodiazepine. Solche Diskrepanzen in den Studienergebnissen sind möglicherweise auf ein unterschiedliches Niveau der zuvor ambulant verordneten Medikamente zurückzuführen.

Auf Basis der Dokumentation einer Allgemeinpraxis untersuchten Himmel et al. (1996b) die Änderungen der Dauermedikation von Patienten, die ins Krankenhaus eingewiesen wurden. Der Prozentsatz der abgesetzten oder geänderten Pharmaka schwankte in den einzelnen Krankenhäusern zwischen 41\% und 69\%. Abgesetzt wurden 28\% der Medikamente (vor allem vasoaktive Substanzen), 17\% wurden durch andere chemische Substanzen oder durch Präparate anderer Hersteller ersetzt.

In einer finnischen Studie über die Gründe für die Überweisung in ein Krankenhaus wurde festgestellt, daß die Klinikärzte bei $42 \%$ der Patienten die Medikation 
änderten. 38\% der Patienten erhielten neue Medikamente, bei jedem zehnten Patienten wurden ein oder mehrere Medikamente abgesetzt (Haiko et al. 1995).

Zwischen den einzelnen medizinischen Fachgebieten und deren Verschreibungspraktiken gibt es zum Teil gravierende Unterschiede. In einer Untersuchung an einer deutschen Universitätsklinik übernahmen nach eigener Aussage weit mehr Chirurgen (82\%) als Internisten (25\%) die Hausarztmedikation (Himmel et al. 1996a). Auch bei der Verordnung von Kombinationspräparaten zeigen die Internisten im Gegensatz zu ihren chirurgisch tätigen Kollegen (82 vs. 41\%) eine kritischere Haltung. Die Autoren begründeten dies einerseits mit den klinischpharmakologischen Standards, an denen sich hauptsächlich Internisten orientieren, andererseits mit der höheren Bedeutung der Pharmakotherapie im klinischen Alltag von Internisten.

An der Frankfurter Universitätsklinik untersuchten Harder et al. (1991) die Verteilung von Sonderanforderungen auf die verschiedenen Kliniken und die Gründe dafür. In den einzelnen Fachgebieten zeigten sich dabei erhebliche Unterschiede im Anforderungsverhalten. So entfielen auf die Orthopädie 29\%, die Innere Klinik $16 \%$, die Neurologie 15\% und die Dermatologie 13\% sämtlicher Sonderanforderungen. Die Mehrzahl der Sonderanforderungen (90\%) war durch die Weiterführung hausärztlicher Verordnungen bedingt.

Mehrere Untersuchungen zum Verordnungsverhalten von Klinikärzten beleuchten auch die Risiken der Polymedikation. Schon bei der gleichzeitigen Einnahme von 3 Medikamenten steigt die Gefahr von unerwünschten Nebenwirkungen auf über 5\% (Nolan und O'Malley 1988). Besonders davon betroffen sind Patienten im höheren Lebensalter, deren prozentualer Anteil an der Gesamtbevölkerung ständig steigt.

Statistisch gesehen erhält in Deutschland jeder Patient über 60 Jahre drei rezeptpflichtige Medikamente, 96\% aller über 70jährigen nehmen sogar durchschnittlich sechs Medikamente pro Tag ein. 
Die Polymorbidität der Patienten erschwert häufig die Einschätzung von unerwünschten Wirkungen einer Medikation, da alterstypische Beeinträchtigungen und unterschiedliche pathologische Veränderungen das klinische Bild verschleiern (Lüttje und Wiesehahn 1997).

McLeod et al. (1997) untersuchten Risiko und Nutzen der Pharmakotherapie bei Patienten im hohen Lebensalter. Anhand einer 4-Punkteskala (unwichtig bis bedeutend) beurteilten 32 Spezialisten (Pharmakologie, Geriatrie, Allgemeinmedizin, klinische Pharmakologie) eine Liste mit 71 pharmakologischen Therapieschemata, werteten deren mögliche Risiken und nannten Behandlungsalternativen. Mehr als die Hälfte der in dieser Studie erfaßten Patienten erhielt Medikamente, die mit einem zu hohen Risiko im Verhältnis zum Nutzen behaftet waren.

Zu einem ähnlichen Ergebnis kamen Bonetti et al. (2000). Die Hälfte der Patienten einer internistischen Station einer Schweizer Klinik erhielt bei der Entlassung Medikamente mit erhöhtem Potential für gefährliche Wechselwirkungen. Die Autoren stellten weiterhin fest, daß die Häufigkeit solcher potentiell gefährlichen Kombinationen mit dem Alter der Patienten steigt.

Bewohner von Pflegeheimen erhalten bedeutend mehr Medikamente als andere Patienten. In einer geriatrischen Klinik wurden Medikamentenverschreibungen bei Aufnahme, bei Entlassung und 18 Monate nach Entlassung kontrolliert und verändert (Kruse et al. 1991). Vielverschreibungen (5 oder mehr Medikamente) konnten von $43 \%$ auf $17 \%$ reduziert werden. Durch Vereinfachung der Einnahmeempfehlungen wurde ein Rückgang der Medikamente von durchschnittlich 6,7 bei Aufnahme auf 4,4 Medikamente bei Entlassung erreicht. Kardiovaskuläre Substanzen, Diuretika und Psychopharmaka waren mit $64 \%$ die am häufigsten verschriebenen Medikamente. 
Die Anwendung von Herzglykosiden konnte von 60\% auf 33\% nahezu halbiert werden. 3 Monate nach Entlassung der Patienten aus dem Krankenhaus ähnelte die Verschreibung jedoch wieder der Ausgangssituation.

Das Ausmaß der Polymedikation ist aufgrund ungenauer Dokumentation sowie zusätzlicher Selbstmedikation seitens der Patienten den Ärzten manchmal gar nicht bewußt. Ärzten müßte die aktuelle Medikation eines Patienten bekannt sein, um Gefahren durch unerwünschte Wechselwirkungen ausschließen zu können. Nicht immer ist dies gewährleistet. In ihrer Studie wollten Claoué und Elkington (1986) herausfinden, wie genau britische Allgemeinärzte über die Medikation ihrer Patienten informiert sind. Die Diskrepanzen zwischen dem, was die Patienten offenbar einnahmen, und der ärztlichen Dokumentation waren zum Teil erheblich: In weniger als der Hälfte der Fälle stimmten die Angaben von Arzt und Patienten überein. Zu ähnlichen Ergebnissen kommt eine italienische Studie. Über 40\% der von Spagnoli et al. (1989) untersuchten Patienten nahmen mindestens ein dem Hausarzt unbekanntes Medikament ein. In diesen Fällen wird auch eine Medikamenten-Dokumentation der Hausärzte bei Einweisung eines Patienten lückenhaft sein.

Insbesondere Benzodiazepine werden nach Surendrakumar et al. (1992) zu Beginn einer stationären Behandlung gehäuft verordnet. Die Hälfte der 58 Patienten ihrer Beobachtungsstudie begann im Krankenhaus Benzodiazepine einzunehmen; 12 von ihnen hatten dies schon bei vorangegangenen Klinikaufenthalten getan. In 22 Fällen wurde die Verschreibung nicht dokumentiert, wodurch das Risiko von Mehrfachverordnungen und unerwünschten Nebenwirkungen steigt. Im Gegensatz dazu kommen Himmel et al. (1996a) zu dem Ergebnis, daß die Patienten ihrer Studie nicht mehr Benzodiazepine im Krankenhaus einnahmen als vorher. Dies kann einerseits an der öffentlichen Diskussion über mögliche Abhängigkeitsbildung, andererseits an einer lückenhaften ärztlichen Dokumentation gelegen haben. 


\subsection{Verordnungsverhalten von Hausärzten}

Nach wie vor steht das Verordnungsverhalten der Allgemeinärzte in der Kritik. Ihnen wird vorgeworfen, daß sie zu oft unwirksame oder unnötige Medikamente verschreiben (Tamblyn et al. 1994) und sie damit unter anderem auch die Risiken von Neben- oder Wechselwirkungen für den Patienten erhöhen (Lüttje und Wiesehahn 1997).

Das Verordnungsverhalten der Hausärzte wird von unterschiedlichsten Faktoren beeinflußt. Die Arbeit von Webb und Lloyd (1994) verdeutlicht, wie eng die Handlungen der Hausärzte mit den Patientenwünschen verknüpft sind. 51\% der befragten Patienten erwarteten und 55\% erhielten ein Arzneirezept von ihrem Hausarzt. $13 \%$ hofften auf eine Einweisung in ein Krankenhaus, 10\% wurden eingewiesen. Die Autoren werteten dies als Hinweis dafür, daß Ängste und Hoffnungen der Patienten die Entscheidungen des niedergelassenen Arztes stark beeinflussen.

Doch auch bei der Übernahme von Patienten wird die Medikation der vorhergehenden Ärzte oft unkritisch übernommen (Dybwad et al. 1997).

Einen großen Einfluß auf die Pharmakotherapie haben ökonomische Rahmenbedingungen. In Deutschland änderte sich besonders durch die Gesundheitsreform 1993 das Verordnungsverhalten der Allgemeinärzte nachhaltig. Das strenge Medikamentenbudget zwang Ärzte, sich kritisch mit den Kosten der Behandlungsstrategien auseinanderzusetzen und diese gegebenenfalls zu ändern. Nicht wenige Ärzte sahen darin zugleich eine Einschränkung der bisher erfolgreichen Behandlungen (Hoopmann et al. 1995). Himmel et al. (1997) stellten gleichfalls fest, daß auf Grund der Gesundheitsreform 60\% der Allgemeinärzte ihr Verschreibungsverhalten änderten. Sie verwendeten häufiger kostengünstigere Generika und zeigten sich Patientenwünschen gegenüber weniger liberal.

Nicht in jedem Fall ist der Hausarzt für die verschriebenen Medikamente verantwortlich. In einer spanischen Studie untersuchte Callol (1999) den Einfluß von 
Fremdverschreibungen auf die Hausarztmedikation. Von 5427 Verschreibungen entfiel ca. die Hälfte auf Selbstmedikation bzw. wurde durch Krankenhäuser, Privatpraxen und Fachärzte verursacht. Auch Jones MI et al. (2001) beschreiben in ihrer Studie Probleme mit Fremdverschreibungen. Die Übernahme von im Krankenhaus verordneten Medikamenten stellt englische Allgemeinärzte vor eine schwierige Situation. Durch diese Arzneimittel wird das Budget der Praxen zusätzlich belastet und bringt die Ärzte häufig in Konfliktsituationen, da das Absetzen oder Verändern der Krankenhausmedikamente das Verhältnis zwischen Arzt und Patient empfindlich stören kann. Ebenso wird die Zusammenarbeit der Ärzte im ambulanten und stationären Bereich durch Veränderung der Krankenhausmedikation negativ beeinflußt.

Als nicht optimal bezeichneten Coste und Venot (1999) das Verordnungsverhalten französischer Allgemeinärzte. Bei einer landesweiten Analyse stellten sie fest, daß zwischen $32 \%$ und $88 \%$ der von Allgemeinärzten verordneten Medikamente ineffektiv und die Risiken für Arzneimittel-Wechselwirkungen und Überdosierungen erhöht waren. Die Studie macht auf die Vielschichtigkeit und Komplexität primärärztlichen Verordnungsverhaltens aufmerksam. Nach Meinung der Autoren könnten Weiterbildungsprogramme die Sicherheit für Patient und Arzt erhöhen und gleichzeitig die Kosteneffizienz in den Praxen steigern.

Bateman et al. (1996) halten wissenschaftliche Arbeitsgruppen für geeignet, um Kriterien und Standards für eine qualitativ höherwertige Arzneitherapie zu entwickeln und in der Praxis einzusetzen. Schon relativ einfache Weiterbildungsmaßnahmen könnten das Wissen der Ärzte über Kosten und Wirksamkeit von Medikamenten verbessern (Frazier et al. 1991).

In England wurden 13 Kriterien für eine pharmakologische Langzeitbehandlung entwickelt, die in einer Allgemeinpraxis erprobt wurden. Neun dieser Kriterien wurden als geeignet eingestuft, jedoch die Problematik und Schwierigkeit einer Beurteilung unterstrichen (Cantrill et al. 1998). 
McGavock et al. (1994) konnten mit Hilfe von COMPASS, einer monatlichen Online-Verschreibungsanalyse, Allgemeinärzten für ihre Praxis Einsparungsmöglichkeiten und Verschreibungsvorschläge unterbreiten. Schwerpunkte des COMPASS-Berichtes waren:

1. Rangliste und Häufigkeit der verschriebenen Medikamente

(im Vergleich zum Fachgruppendurchschnitt; exzessive Werte sollten abschrecken)

2. Nicht eindeutige bzw. inkorrekte Verschreibungen (z.B. Antibiotika)

3. Voraussagen von Verschreibungskosten

(basierend auf praxisdemographischen Werten)

4. Verwendung von Medikamenten mit sehr geringer Praxisrelevanz

(z.B. periphere Vasodilatatoren)

Die nordirischen Allgemeinärzte empfanden COMPASS als äußerst wertvoll - in Hinblick auf Kosteneffektivität und Verschreibungsqualität.

In einer schwedischen Region befragten Tomson et al. (1994) 125 Allgemeinärzte, wie wichtig die Arzneimittelliste der Universitätsklinik für ihre tägliche Arbeit sei. In der Folge entwickelten die Ärzte ihrerseits in Seminaren eine Medikamentenliste mit 167 Empfehlungen. Nach kurzer Anwendungszeit nahmen Umfang und Kosten der Verordnungen eindeutig ab. Die Medikamentenkosten lagen 20\% unter dem Landesdurchschnitt. Durch die im Rahmen der Studie notwendig gewordene Zusammenarbeit zwischen Kliniker und Allgemeinarzt konnte auch die Kluft zwischen Krankenhaus und Allgemeinpraxis geschlossen werden.

Zu einer signifikanten Reduzierung des Medikamentenumfangs in allgemeinmedizinischen amerikanischen Praxen führte eine Studie von Fillit et al. (1999). Bei der Überprüfung von über 37.000 Patienten wurde bei $15 \%$ ein potentielles Risiko durch Polymedikation festgestellt. Diesen Patienten empfahl man gemeinsam mit ihrem behandelnden Arzt ein Medikamenten-Review. Den Allgemeinärzten standen dafür Krankenhausrichtlinien zur Polymedikation zur Verfügung. Im Ergebnis wurden bei $20 \%$ der Patienten Medikamente abgesetzt, bei $29 \%$ änderte sich die 
Dosis und 17\% informierten ihren Arzt über Medikamente, die sie ohne sein Wissen nahmen.

Ziel einer Studie von Bernatzky et al. (1999) war es, den gegenwärtigen Stand der Verschreibungsgewohnheiten österreichischer Allgemeinärzte am Beispiel der Schmerztherapie aufzuzeigen. Allerdings beteiligten sich nur 16\% der über 5000 um Mitarbeit gebetenen Ärzte an der Befragung. Knapp 90\% dieser Ärzte kannten das WHO-Stufenschema und verschrieben bei Bedarf auch starke Opioide. Würde man dieses Ergebnis verallgemeinern, wäre die Schmerztherapie österreichischer Ärzte optimal. Bernatzky vermutet allerdings erhebliche Defizite in der Schmerztherapie bei den Ärzten, die nicht geantwortet haben.

\subsection{Probleme der Patienten}

Unter dem Begriff "Compliance" versteht man den Grad, in dem das Verhalten einer Person z.B. in bezug auf die Einnahme eines Medikamentes oder die Durchführung einer Diät mit dem ärztlichen oder gesundheitlichen Rat korrespondiert. In etwas autoritativer Übersetzung wird auch von Folgebereitschaft gesprochen (Haynes 1982). Um eine optimale Therapie erreichen zu können, ist die Mitarbeit des Patienten unerläßlich. Bedeutende Faktoren der Compliance sind die Person des Arztes, der Patient und die Therapie, wobei der Arzt den größten Einfluß ausübt, zumal er die Therapie gestalten kann und traditionell den Patienten leitet (O'Hanrahan und O'Malley 1981).

Laut Stevenson et al. (2000) ist das traditionelle Modell der medizinischen Entscheidungsfindung, bei der der Arzt die Entscheidung zum Wohl des Patienten trifft, zunehmend veraltet. Neue Modelle beziehen den Patienten aktiv in die Entscheidung über seine Behandlung ein. Beispielsweise stützen sich Charles et al. (1997) auf 4 Hauptkriterien:

1. Arzt und Patient sind beteiligt

2. Arzt und Patient informieren sich gegenseitig 
3. beide Parteien entscheiden über die bevorzugte Behandlung

4. um eine Behandlung durchzuführen, muß eine Einigung erfolgt sein.

Vor allem die ersten beiden Punkte sind nach Meinung der Autoren der Schlüssel für die neue Art der Zusammenarbeit zwischen Arzt und Patient.

Mißverständnisse zwischen Arzt und Patient können zur ablehnenden Haltung der Patienten gegenüber therapeutischen Maßnahmen führen, wenn z.B. der Arzt nur mangelhaft über die Patienten informiert ist oder die Patienten die ärztlichen Entscheidungen nicht kennen bzw. verstehen.

Viele dieser Mißverständnisse resultieren auch aus der mangelhaften Beteiligung der Patienten am Konsultationsgespräch. So äußern Patienten vergleichsweise selten Hoffnungen und Wünsche sowie Meinungen zu ärztlichen Entscheidungen (Britten und Ukoumunne 1997).

Die Pharmakotherapie ist ein wesentlicher Bestandteil allgemeinärztlicher Behandlung. Laut Kochen (1998) nehmen jedoch durchschnittlich 50\% aller Patienten ihre Medikamente nicht richtig, unregelmäßig oder überhaupt nicht ein. Dowell und Hudson (1997) befragten 50 allgemeinmedizinisch betreute Patienten zu ihrem Umgang mit Medikamenten und faßten die Ergebnisse im folgenden Modell zusammen:

1. Das Wissen der Patienten über Erkrankung und Behandlung, kombiniert mit Vertrauen zum Arzt, erhöht die Motivation zur Medikamenteneinnahme.

2. Die meisten Patienten "testen“ ein Medikament, bevor sie es akzeptieren.

3. Bei der Medikamenteneinnahme lassen sich 3 Typen von Patienten finden:

- den passiv Einnehmenden (weil der Doktor es wünscht)

- den aktiv Einnehmenden (sie entscheiden selbst, ob und wann sie etwas einnehmen)

- den generell nicht Einnehmenden.

4. Der Prozeß, die Behandlung zu akzeptieren, ist eng mit dem Prozeß verknüpft, die Krankheit zu akzeptieren.

5. Technische Einnahmeprobleme spielen nur eine untergeordnete Rolle. 
Obwohl die Mitarbeit der Patienten äußerst wichtig für eine erfolgreiche Pharmakotherapie ist, sind die Auskünfte der Patienten über ihre aktuelle Medikamenteneinnahme oftmals ungenau (Van Hessen et al.1990). Durch selbstständige Änderungen der Einnahmevorschriften kommt es auch zu Mehrfach- und Falscheinnahmen von Arzneimitteln, über die die behandelnden Ärzte wiederum in der Regel nicht informiert sind.

Mahdy und Seymour (1990) befragten 261 Patienten eines Pflegedienstes detailliert über deren Medikamente. Die Fragen wurden hierzu in 4 Kategorien eingeteilt:

- Medikamentenname

- Dosierung

- Einnahmehäufigkeit

- Grund der Verschreibung.

Die Auskünfte fast aller Befragten wurden als mangelhaft eingestuft. Nur ca. 10\% der Patienten konnten die verschriebenen Medikamente vollständig benennen. Im Gegensatz zu den ambulant betreuten Patienten machten die stationär aufgenommenen Patienten deutlich mehr Fehler. Die Anzahl der Fehler korrelierte mit steigendem Alter, sinkender geistiger Leistungsfähigkeit sowie der Zahl der verschriebenen Medikamente.

Durch eine Befragung in einem Altenheim wollten Torrible und Hogan (1997) herausfinden, wie gut Ärzte und Pharmakologen über die Medikation ihrer Patienten informiert waren. Die Patienten nahmen nicht nur zusätzlich rezeptfreie Arzneien ein, sondern verfügten auch über ein beachtliches Lager an bereits abgesetzten Medikamenten. Die behandelnden Ärzte und Pharmakologen wußten nur sehr wenig über den Arzneimittelverbrauch der Heimbewohner. Die Autoren empfehlen daher Ärzten eine sorgfältige Überprüfung der Medikation ihrer Patienten sowie ihres aktuellen Einnahmeverhaltens.

Eine entscheidende Rolle in der Patientencompliance spielt die Anzahl der verordneten Medikamente. Laut Lüttje und Wiesenhahn (1997) liegt die Compliance für ein Medikament relativ konstant bei $80-85 \%$. Bei gleichzeitiger Gabe von 5 
Medikamenten sinke sie jedoch auf ca. 33-40\% ab. Spagnoli et al. (1989) registrierten eine Verschlechterung der Patientencompliance bereits dann, wenn die Medikamente von einem Krankenhausarzt und nicht vom Hausarzt verschrieben werden.

Die Umstellung einer Langzeitmedikation, sei es durch Ab- oder Ansetzen bzw. durch Wechsel eines Präparates, stellt in jedem Fall einen Eingriff in die Gewohnheiten des Patienten dar, wodurch es zu mannigfaltigen Problemen kommen kann (Green et al. 1985).

$\mathrm{Daß}$ Patienten jedoch häufig über Veränderungen in der Arzneimitteltherapie im Unklaren gelassen werden, verdeutlichen Himmel et al. (1996b): Nur ca. 50\% der stationär aufgenommenen Patienten konnten sich daran erinnern, über Änderungen der Medikation unterrichtet worden zu sein. Eine gezielte Patientenaufklärung über Änderungen der Medikation ist laut Cochrane et al. (1992) für die Compliance von größter Bedeutung. Etwa 90\% der von innen untersuchten 50 geriatrischen Patienten hatten nach einem Zeitraum von 2 Wochen einen anderen Einnahmemodus als im Entlassungsbrief des Krankenhauses vorgeschlagen. $11 \mathrm{~Pa}-$ tienten änderten die Dosis, 20 Patienten nahmen Medikamente, die nicht empfohlen wurden, und 10 Patienten setzten die Medikation einfach ab.

Auch Vollmann und Helmchen halten die genaue Aufklärung über Diagnose und Therapie sowie die Einwilligung des Patienten in ärztliche Handlungen für unerläßlich für eine gute Patientencompliance. Zeitmangel und Unverständlichkeit bezeichnen Patienten als häufigste Mängel (70-80\%) ärztlicher Aufklärungsgespräche. Die Qualität der Arzt-Patient-Beziehung, die Art und Weise der Informationsvermittlung und die Gefährlichkeit der Erkrankung bzw. der Behandlung werden als ausschlaggebend für die Patientenzufriedenheit angesehen (Vollmann und Helmchen 1997).

Nicht alle Patienten sind gleichermaßen an einer Aufklärung über ihre Medikation interessiert. Schmeling-Kludas et al. (1991) befragten 56 internistische und 62 chirurgische Patienten zu psychosozialen Belastungen nach Krankenhausauf- 
nahme. Ältere Patienten (> 60. Lebensjahr) hatten geringeres Interesse an einer Aufklärung über therapeutische Maßnahmen. Diese Patientengruppe wurde oftmals durch das Pflegepersonal informiert. In einer ähnlich angelegten Arbeit zeigten sich stationär eingewiesene Patienten in 16\% der Fälle als sehr gut, in $49 \%$ gut, in $21 \%$ mittelmäßig oder gering und in $2 \%$ als gar nicht über Diagnose und Therapiemaßnahmen informiert (Schmeling-Kludas et al. 1989).

Dowell et al.(1995) untersuchten, ob vermehrter Generikaeinsatz in den Allgemeinpraxen zu finanzieller Entlastung führt, ohne die Zufriedenheit der Patienten durch die Umstellung zu berühren. Vier Monate nach Medikationsänderung wurden 280 Patienten nach ihren Erfahrungen befragt. Trotz ausführlicher ArztPatientengespräche resultierten aus 100 Medikationsänderungen 16 zusätzliche Arztbesuche. Ca. 45\% der Patienten waren mehr oder weniger stark unzufrieden über die Eingriffe in ihre Medikation, woran auch weitere Gespräche nichts ändern konnten. Offensichtlich reagieren einige Patienten selbst auf pharmakologisch geringfügige Veränderungen der Medikation hochsensibel. 


\section{Fragestellung und Hypothesen der Untersuchung}

Bei der stationären Aufnahme eines Patienten in ein Krankenhaus wird die hausärztliche Medikation oft verändert. Ziel der vorliegenden Arbeit ist es, diese Veränderungen der hausärztlichen Arzneimitteltherapie in kommunalen Krankenhäusern nachzuzeichnen. Die detaillierten Gründe für die Umstellung der Arzneimitteltherapie und ihre Angemessenheit können dabei nicht erhoben und überprüft werden.

Defizite in der Patientenaufklärung über Ursachen, Verlauf und Bewältigung von Krankheiten wurden oft im ambulanten und stationären Bereich beschrieben. Inhalt und Qualität der Medikamenten-Aufklärung durch den Krankenhausarzt, speziell das Wissen der Patienten über die neu verordneten Medikamente bilden den zweiten Studienschwerpunkt der Arbeit.

Basierend auf dem Forschungsstand wurden folgende Hypothesen entwickelt:

\section{Hypothese}

Über die Hälfte der hausärztlich verordneten Medikamente werden in den Krankenhäusern geändert.

Kommentar: Die medikamentöse Behandlung von Krankheiten ist gerade in der Inneren Medizin eine wichtige Therapieoption. Bei Krankenhauseinweisung eines hausärztlich betreuten Patienten wird die bis dato erfolgte Arzneimitteltherapie nicht selten in Frage gestellt und teils gravierend verändert. In der Literatur gibt es widersprüchliche Ergebnisse zum Umfang der Veränderungen hausärztlicher Medikation durch Klinikärzte. 


\section{Hypothese}

Antihypertensiva und Kardiovaskuläre Substanzen sind in den Krankenhäusern am häufigsten von Änderungen betroffen.

Kommentar: Herz-Kreislauferkrankungen und Bluthochdruck zählen in unserer Gesellschaft zu den häufigsten Erkrankungen. Der Anteil an Antihypertensiva und kardiovaskulären Substanzen in der Einweisungsmedikation ist dementsprechend hoch, besonders bei Einweisungen in internistische Abteilungen. Da gerade Internisten häufig die Einweisungsmedikation umstellen, dürften in den beiden Substanzklassen Änderungen häufig sein.

\section{Hypothese}

Krankenhausärzte verschreiben deutlich weniger Generika als Hausärzte.

Kommentar: Seit der Gesundheitsreform müssen Ärzte noch ökonomischer arbeiten, z.B. durch häufige Verordnung kostengünstiger Generika. Auch in den Krankenhäusern führt wirtschaftliche Druck zu Veränderungen in der täglichen Verordnungspraxis. Allerdings betrifft der Sparzwang den Klinikarzt nicht unmittelbar, so daß nicht von einem rapiden und zügigem Umstieg auf kostengünstige Medikamente auszugehen ist. Eine Diskrepanz im Generikaeinsatz zwischen Klinik und Praxis ist somit zu erwarten. 


\section{Hypothese}

Kommunale Krankenhäuser ändern die Hausarztmedikation seltener als Universitätskliniken.

Kommentar: Kreiskrankenhäuser haben in der Regel ein relativ begrenztes Versorgungsgebiet mit einer überschaubaren Anzahl einweisender Hausärzte. Nicht selten besteht zwischen hausärztlich und stationär tätigen Ärzten ein langjähriges Arbeits- und Vertrauensverhältnis. Universitätskliniken hingegen arbeiten überregional und weitestgehend losgelöst vom Hausarzt. Sie verfügen über eine große Anzahl an Spezialisten und Wissenschaftlern, was insgesamt einen kritischen Umgang mit der Hausarztmedikation vermuten läßt.

\section{Hypothese}

Patienten wissen nach der Aufklärung über Veränderungen in der Arzneimitteltherapie nicht entscheidend mehr als vorher.

Kommentar: Jede Umstellung der Langzeitmedikation erfordert eine detaillierte Aufklärung der Patienten über Art und Umfang der Veränderungen. Jedoch werden Patienten nach Krankenhauseinweisung häufig nur ungenügend über Veränderungen ihrer Medikation informiert.

\section{Hypothese}

Jüngere Patienten werden häufiger durch den Krankenhausarzt über Medikationsänderungen aufgeklärt.

Kommentar Mit zunehmenden Alter sinkt das Interesse der Patienten an einem Aufklärungsgespräch über therapeutische Maßnahmen. Es gibt vergleichsweise 
wenig Studien über die Patientenaufklärung nach Krankenhauseinweisung. Die Ergebnisse von Befragungen in Pflegeheimen und Hausarztpraxen sind nur bedingt auf Krankenhäuser übertragbar. Diese Arbeit in Kliniken gleicher Kategorie untersucht die Aufklärungsgewohnheiten der Ärzte und das Wissen der Patienten - nach Altersstufen - während des stationären Aufenthaltes.

\section{Material und Methoden}

Die Daten für diese Arbeit wurden auf den internistischen Stationen in 3 Kreiskrankenhäusern im Zeitraum von Mitte Juli bis Ende Oktober erhoben. Im Juni desselben Jahres wurde in jedem Krankenhaus eine Pilotstudie durchgeführt, die jedoch keine Änderungen der Dokumentationsstruktur erforderlich machte.

\subsection{Krankenhäuser}

Die Studie wurde in 3 Krankenhäusern gleicher Kategorie (Kreiskrankenhaus) und Größe (je Haus ca. 200-300 Betten) durchgeführt. In den einzelnen Häusern selbst fand die Erhebung auf rein internistischen Stationen statt (ca. 80 bis 100 internistische Betten je Haus) - mit Behandlungsschwerpunkten in Gastroenterologie und Kardiologie.

Die Kliniken befinden sich in Nordthüringen innerhalb eines Landkreises bzw. in einem angrenzenden Landkreis, wobei gleiche infrastrukturelle Rahmenbedingungen vorlagen. Im Einzugsgebiet der Krankenhäuser und der niedergelassenen Praxen sind, soweit feststellbar, bisher keine oder kaum wissenschaftliche Studien durchgeführt worden. 


\subsection{Patienten}

Die Auswahl der Patienten erfolgte zufällig: Aufgenommen wurden alle an den Erhebungstagen auf der Station befindlichen Patienten, die folgende Auswahlkriterien erfüllten:

- internistischer Patient

- Volljährigkeit

- ausreichende Deutschkenntnisse

- Langzeitmedikation durch den Hausarzt (mindestens 2 Medikamente)

- Mindestkrankenhausaufenthalt zum Erhebungszeitpunkt: 2 Tage

\subsection{Datenerhebung}

Aus den Krankenakten der Patienten bzw. per Interview wurden folgende Daten erhoben:

- Alter

- Geschlecht

- Beruf/ Bildung

- Vorerkrankungen

- Hausarzt- und Einweisungsdiagnosen

- Klinikdiagnosen

- Praxismedikation

Anhand des Einweisungsbriefes und der Anamnese wurden alle bis zum Zeitpunkt der stationären Aufnahme verordneten Medikamente dokumentiert.

- Stationäre Medikation

Durch Einsicht in die jeweiligen Krankenblätter wurde die stationäre Arzneimittelverordnung festgehalten. 


\subsection{Patientenbefragung}

Alle 300 Patienten wurden anhand eines standardisierten Fragebogens (s. Anhang) interviewt, sofern sie einverstanden und gesundheitlich dazu in der Lage waren. Die Befragung erfolgte nach Einsicht in die jeweilige Patientenakte. Über den gesamten Untersuchungszeitraum wurde das Interview von derselben Person durchgeführt.

\subsection{Datenaufbereitung und - Auswertung}

Für die bessere Auswertung der Daten wurden die Patienten in Altersgruppen eingeteilt:

- Altersgruppe $1=$ Alter zwischen 19 und 34 Jahren

- Altersgruppe 2 = Alter zwischen 35 und 49 Jahren

- Altersgruppe $3=$ Alter zwischen 52 und 64 Jahren

- Altersgruppe $4=$ Alter zwischen 65 und 79 Jahren

- Altersgruppe $5=$ Alter zwischen 80 und 97 Jahren

Für den Vergleich der Medikamente aus Praxis und Krankenhaus bzw. deren Änderungen gelten folgende Definitionen:

- Keine Änderung

Substanz, Handelsname und Dosierung sind identisch

- Absetzen von Medikamenten

Arzneimittel, die nach stationärer Aufnahme nicht mehr verabreicht wurden

- Medikamentöse Umstellungen

Präparate anderer Hersteller (anderer Handelsname)

- Neue Medikamente

Alle stationär neu angesetzten Medikamente

- Dosisänderungen

Alle stationär erfolgten Dosisänderungen 
Um eine Gruppierung und statistische Auswertung der verordneten Medikamente vornehmen zu können, wurden alle dokumentierten Arzneimittel nach der Anatomisch-therapeutischen-chemischen Klassifikation für den deutschen Arzneimittelmarkt (ATC-Code, Stand 1998) codiert.

Die Berechnung der Daten beschränkte sich überwiegend auf univariate und bivariate Auswertungen mit absoluten und relativen Häufigkeitsangaben. Alle Auswertungen erfolgten mit dem Statistikprogramm SAS.

\section{Ergebnisse}

\subsection{Patienten}

Das Durchschnittsalter der männlichen Patienten war 67,7 Jahre (Range: 31 bis 86). Die weiblichen Patienten waren im Mittel 72 Jahre (Range: 19 - 97). In den Krankenhäusern 1 und 2 waren die befragten Patienten etwas älter als im Krankenhaus 3. Da es sich um internistische Abteilungen mit den Schwerpunkten Kardiologie und Gastroenterologie handelte, war der Anteil älterer Patienten erwartungsgemäß hoch. Zum Zeitpunkt der Befragung waren die Patienten in den Häusern 1 und 3 ca. 8 Tagen stationär aufgenommen. Im Krankenhaus 2 waren die Patienten seit durchschnittlich 6,5 Tage in stationärer Behandlung (Tabelle1).

Von den 300 Patienten waren 59\% Frauen. Über die Hälfte der untersuchten Patienten ( $\mathrm{N}=173$ ) waren in der Altersgruppe zwischen 65-79 Jahren und lediglich 6,3\% waren im Erhebungszeitraum jünger als 50 Jahre (Tabelle 2). 
Tabelle 1: Zahl, Alter und Krankenhausaufenthalt der Patienten

\begin{tabular}{ccccccc}
\hline $\begin{array}{c}\text { KRANKEN- } \\
\text { HAUS }\end{array}$ & PATIENTEN & \multicolumn{2}{c}{$\begin{array}{c}\text { ALTER } \\
\text { (in Jahren) }\end{array}$} & \multicolumn{3}{c}{$\begin{array}{c}\text { KH-AUFENTHALT } \\
\text { (in Tagen) }\end{array}$} \\
& (N) & \multicolumn{1}{c}{$\overline{\mathbf{x}}$} & (SD) & \multicolumn{1}{c}{$\overline{\mathbf{x}}$} & (SD) \\
\hline $\mathbf{1}$ & 100 & 73,4 & $(8,6)$ & 7,9 & $(4,0)$ \\
$\mathbf{2}$ & 100 & 67,4 & $(13,6)$ & 6,5 & $(2,9)$ \\
$\mathbf{3}$ & 100 & 71,1 & $(12,2)$ & 7,9 & $(3,8)$ \\
\hline
\end{tabular}

Tabelle 2: Verteilung der Geschlechter in den Altersgruppen

\begin{tabular}{|c|c|c|c|c|c|}
\hline \multirow[b]{2}{*}{ ALTERSGRUPPE } & \multicolumn{4}{|c|}{ Geschlecht } & \multirow{2}{*}{$\begin{array}{c}\text { Summe } \\
\text { (N) }\end{array}$} \\
\hline & M & $(\%)$ & w & $(\%)$ & \\
\hline 1 (19 - 34 Jahre) & 2 & $(40)$ & 3 & (60) & 5 \\
\hline 2 (35 - 49 Jahre) & 9 & (64) & 5 & (36) & 14 \\
\hline 3 (52 - 64 Jahre) & 29 & (57) & 22 & (43) & 51 \\
\hline 4 (65 - 79 Jahre) & 67 & (38) & 107 & (62) & 174 \\
\hline 5 (80 - 97 Jahre) & 16 & (29) & 40 & (71) & 56 \\
\hline Gesamt & 123 & $(41 \%)$ & 177 & $(59 \%)$ & 300 \\
\hline
\end{tabular}

\subsection{Vorerkrankungen, Einweisungs- und Krankenhausdiagnosen}

Die Akten von 300 stationär eingewiesenen Patienten wurden während dieser Studie eingesehen. Dabei sind anhand der Anamnese und des Einweisungsscheines alle Erkrankungen dokumentiert worden, die schon vor Krankenhauseinweisung bestanden (Vorerkrankungen). Ebenso wurden alle Diagnosen 
erfaßt, die zur Einweisung in das Krankenhaus führten (Einweisungsdiagnosen) und diejenigen Diagnosen, die neu im Krankenhaus gestellt wurden (Krankenhausdiagnosen). All diese Erkrankungen wurden in 10 Diagnosegruppen unterteilt (Tabelle 3).

Zu den häufigsten Vorerkrankungen gehörten die des Herz-Kreislauf-Systems gefolgt von den Stoffwechsel-, Ernährungs- und endokrinen Störungen.

Bei den Einweisungsdiagnosen lagen Herz-Kreislauferkrankungen ebenfalls an erster Stelle gefolgt von den Erkrankungen des Verdauungstraktes und der Atemwege.

Auf den internistischen Stationen waren ebenfalls die HerzKreislauferkrankungen und Erkrankungen des Verdauungstraktes am häufigsten dokumentiert.

Tabelle 3: Diagnosen und deren Häufigkeit im Vergleich zwischen Vorerkrankungen Einweisungs- und Krankenhausdiagnosen 


\begin{tabular}{|c|c|c|c|c|c|c|}
\hline \multirow[t]{2}{*}{ Diagnosen } & \multicolumn{2}{|c|}{$\begin{array}{c}\text { Vorer- } \\
\text { krankungen }\end{array}$} & \multicolumn{2}{|c|}{$\begin{array}{l}\text { Einweisungs- } \\
\text { diagnosen }\end{array}$} & \multicolumn{2}{|c|}{$\begin{array}{l}\text { Krankenhaus- } \\
\text { diagnosen }\end{array}$} \\
\hline & $\mathbf{N}$ & (\%) & $\mathbf{N}$ & (\%) & $\mathbf{N}$ & (\%) \\
\hline $\begin{array}{l}\text { Erkrankungen des Herz- } \\
\text { Kreislauf-Gefäß-Systems }\end{array}$ & 345 & (49) & 217 & (48) & 203 & (49) \\
\hline $\begin{array}{l}\text { Ernährungs-Stoffwechsel- } \\
\text { u. endokrine Störungen }\end{array}$ & 135 & (19) & 38 & (9) & 37 & (9) \\
\hline $\begin{array}{l}\text { Erkrankungen des } \\
\text { Verdauungstraktes }\end{array}$ & 72 & (10) & 65 & (14) & 59 & (15) \\
\hline $\begin{array}{l}\text { Erkrankungen des } \\
\text { Respirationstraktes }\end{array}$ & 45 & (6) & 62 & (13) & 57 & (14) \\
\hline $\begin{array}{l}\text { Urologische } \\
\text { Erkrankungen }\end{array}$ & 39 & (5) & 17 & (4) & 9 & (2) \\
\hline $\begin{array}{l}\text { Erkrankungen des } \\
\text { Muskel-u. Skelettsystems }\end{array}$ & 23 & (4) & 3 & $(0,5)$ & 3 & $(0.7)$ \\
\hline $\begin{array}{l}\text { Neurologische u. } \\
\text { Psychiatrische Erkr. }\end{array}$ & 16 & (2) & 8 & (2) & 11 & (3) \\
\hline $\begin{array}{l}\text { Gynäkologische Erkran- } \\
\text { kungen u. Erkr. } \\
\text { der Genitalia }\end{array}$ & 16 & (2) & 4 & (1) & 4 & (1) \\
\hline $\begin{array}{l}\text { Hämatologische } \\
\text { Erkrankungen }\end{array}$ & 14 & (2) & 17 & (4) & 18 & $(4,3)$ \\
\hline $\begin{array}{l}\text { Sonstige Erkrankungen } \\
\text { (Synkope, Infektionskr., } \\
\text { Koma, usw.) }\end{array}$ & 4 & (1) & 19 & $(4,5)$ & 10 & (2) \\
\hline GESAMT & 709 & $100 \%$ & 450 & $100 \%$ & 411 & $100 \%$ \\
\hline
\end{tabular}

Betrachtet man die einzelnen Krankenhäuser, sind teils erhebliche Unterschiede in den Diagnosegruppen festzustellen. So sind beispielsweise 92\% aller Erkrankungen des Stoffwechsels und Endokrinums im Krankenhaus 3 lokalisiert. Lediglich bei den Herz-Kreislauferkrankungen erscheint die Verteilung homogen (Tabelle 4).

Tabelle 4: Krankenhausdiagnosen 
KRANKENHAUS

\begin{tabular}{|c|c|c|c|c|c|c|c|c|}
\hline \multirow{2}{*}{$\begin{array}{l}\text { Krankenhaus- } \\
\text { diagnosen }\end{array}$} & \multicolumn{2}{|c|}{ Alle } & \multicolumn{2}{|c|}{1} & \multicolumn{2}{|c|}{2} & \multicolumn{2}{|c|}{3} \\
\hline & $\mathbf{N}$ & (\%) & $\mathbf{N}$ & (\%) & $\mathbf{N}$ & (\%) & $\mathbf{N}$ & (\%) \\
\hline $\begin{array}{l}\text { Erkrankungen des Herz- } \\
\text { Kreislauf-Gefäß-Systems }\end{array}$ & 207 & $(50)$ & 50 & (49) & 87 & (68) & 70 & (37) \\
\hline $\begin{array}{l}\text { Erkrankungen des } \\
\text { Verdauungstraktes }\end{array}$ & 59 & (14) & 2 & (2) & 1 & (1) & 34 & (18) \\
\hline $\begin{array}{l}\text { Erkrankungen des } \\
\text { Respirationstraktes }\end{array}$ & 57 & (14) & 27 & (26) & 22 & (17) & 10 & (6) \\
\hline $\begin{array}{l}\text { Ernährungs-Stoffwechsel- } \\
\text { u. endokrine Störungen }\end{array}$ & 37 & (9) & 5 & (5) & 11 & (8) & 41 & (22) \\
\hline $\begin{array}{l}\text { Hämatologische } \\
\text { Erkrankungen }\end{array}$ & 18 & (4) & 0 & (0) & 1 & (1) & 8 & (5) \\
\hline $\begin{array}{l}\text { Neurologische u. } \\
\text { Psychiatrische Erkr. }\end{array}$ & 11 & (3) & 0 & (0) & 0 & (0) & 3 & (2) \\
\hline $\begin{array}{l}\text { Sonstige Erkrankungen } \\
\text { (Synkope, Infektionskr., } \\
\text { Koma, usw. ) }\end{array}$ & 10 & $(2,5)$ & 0 & (0) & 5 & (4) & 6 & (3) \\
\hline $\begin{array}{l}\text { Urologische } \\
\text { Erkrankungen }\end{array}$ & 9 & (2) & 0 & (0) & 0 & (0) & 4 & (2) \\
\hline $\begin{array}{l}\text { Gynäkologische Erkran- } \\
\text { kungen u. Erkr. } \\
\text { der Genitalia }\end{array}$ & 4 & (1) & 9 & (9) & 0 & (0) & 9 & (5) \\
\hline $\begin{array}{l}\text { Erkrankungen des } \\
\text { Muskel-u. Skelettsystems }\end{array}$ & 3 & $(0,5)$ & 9 & (9) & 1 & (1) & 0 & (0) \\
\hline GESAMT & 411 & $100 \%$ & 103 & $100 \%$ & 130 & $100 \%$ & 188 & $100 \%$ \\
\hline
\end{tabular}

5.3 Hausärztliche Verordnungen 
Zum Zeitpunkt ihrer stationären Aufnahme erhielten die Patienten laut Dokumentation in den Krankenakten 1331 hausärztlich verordnete Medikamente (Durchschnitt: 4,4 Arzneimittel pro Patient). Am häufigsten verordneten die Hausärzte Antihypertensiva und kardiovaskuläre Substanzen (Tabelle 5).

Tabelle 5: Arzeimittelverordnung und deren Häufigkeit in der Praxis

\begin{tabular}{lcc}
\hline Medikamentengruppen $^{\star}$ & Praxismedikation & $\mathbf{( \% )}$ \\
\hline Antihypertensiva & 428 & $(\mathbf{3 2 , 2})$ \\
Kardiovaskuläre Substanzen & 269 & $\mathbf{( 2 0 , 2 )}$ \\
Antidiabetika & 115 & $\mathbf{( 8 , 7 )}$ \\
Antikoagulanzien & 104 & $\mathbf{( 7 , 8 )}$ \\
Substanzen des & 95 & $\mathbf{( 7 , 1 )}$ \\
Respirationstraktes & & \\
Substanzen des ZNS & 81 & $\mathbf{( 6 , 1 )}$ \\
Antirheumatika & 61 & $\mathbf{( 4 , 6 )}$ \\
Substanzen des & 59 & $\mathbf{( 4 , 4 )}$ \\
Verdauungstraktes & & \\
Vitamine & 46 & $\mathbf{( 3 , 5 )}$ \\
Hormone & 43 & $\mathbf{( 3 , 2 )}$ \\
Mineralien & 19 & $\mathbf{( 1 , 4 )}$ \\
Antibiotika & 11 & $\mathbf{( 0 , 8 )}$ \\
\hline GESAMT & $\mathbf{1 3 3 1}$ & $\mathbf{( 1 0 0 \% )}$ \\
\hline
\end{tabular}

( ${ }^{*}$ laut ATC-Code 1998; Mineralien und Vitamine aus der Gruppe „Substanzen des Verdauungstraktes“ wurden zur besseren Übersicht extra gruppiert) 


\subsection{Veränderungen der Hausarztmedikation im Krankenhaus}

Nach der Einweisung kam es bei über der Hälfte der hausärztlich verordneten Medikamente zu Veränderungen; 647 der 1331 Medikamente (48,6\%) übernahmen die Stationen unverändert. Ein Drittel $(36,1 \%)$ setzten die Krankenhausärzte ab. Während des Klinikaufenthaltes erhielten die Patienten 1562 Medikamente. Das waren für jeden Patienten durchschnittlich 5,2 Medikamente im Vergleich zu 4,4 Arzneimittel vor der stationären Einweisung. Krankenhaus 2 setzte deutlich seltener Medikamente ab (Tabelle 6).

Tabelle 6: Veränderungen der Einweisungsmedikation im Krankenhaus

\begin{tabular}{|c|c|c|c|c|c|}
\hline \multirow[b]{3}{*}{ Einweisungsmedikation } & \multicolumn{3}{|c|}{ Krankenhaus } & \multicolumn{2}{|c|}{ Alle } \\
\hline & 1 & 2 & 3 & & \\
\hline & $\mathbf{N}$ & $\mathbf{N}$ & $\mathbf{N}$ & $\mathbf{N}$ & (\%) \\
\hline Medikament abgesetzt & 181 & 101 & 200 & 482 & $(36,1)$ \\
\hline nur Dosis geändert & 42 & 31 & 30 & 103 & $(7,7)$ \\
\hline $\begin{array}{l}\text { gleiche Substanz anderer } \\
\text { Hersteller }\end{array}$ & 8 & 30 & 29 & 67 & $(5,1)$ \\
\hline $\begin{array}{l}\text { gleiche Substanz anderer } \\
\text { Hersteller+Dosis geändert }\end{array}$ & 22 & 8 & 4 & 34 & $(2,5)$ \\
\hline keine Veränderungen & 202 & 234 & 211 & 647 & $(48,6)$ \\
\hline GESAMT & 455 & 405 & 471 & 1331 & $(100 \%)$ \\
\hline
\end{tabular}


Tabelle 7: Veränderungen innerhalb der Medikamentengruppen

\begin{tabular}{|c|c|c|c|c|c|c|}
\hline \multicolumn{7}{|c|}{ VERÄNDERUNG DER PRAXISMEDIKATION IM KRANKENHAUS } \\
\hline \multirow{2}{*}{$\begin{array}{l}\text { Medikamenten- } \\
\text { gruppen* }\end{array}$} & \multirow{2}{*}{$\begin{array}{l}\text { Praxis- } \\
\text { medikamente } \\
\text { N }\end{array}$} & \multicolumn{2}{|c|}{ Abgesetzt } & \multicolumn{2}{|c|}{ Neu } & \multirow{2}{*}{$\begin{array}{c}\text { Gesamt } \\
\text { N }\end{array}$} \\
\hline & & $\mathbf{N}$ & $\%$ & $\mathbf{N}$ & $\%$ & \\
\hline Antihypertensiva & 428 & 128 & (30) & 183 & $(37,9)$ & 483 \\
\hline $\begin{array}{l}\text { Kardiovaskuläre } \\
\text { Substanzen }\end{array}$ & 269 & 106 & (39) & 62 & $(27,5)$ & 225 \\
\hline Antidiabetika & 115 & 37 & (32) & 12 & $(13,3)$ & 90 \\
\hline Antikoagulanzien & 104 & 43 & (41) & 55 & $(47,4)$ & 116 \\
\hline $\begin{array}{l}\text { Substanzen des } \\
\text { Respirationstraktes }\end{array}$ & 95 & 29 & (30) & 88 & $(57,1)$ & 154 \\
\hline Substanzen des ZNS & 81 & 39 & (48) & 94 & $(69,1)$ & 136 \\
\hline Antirheumatika & 61 & 27 & (44) & 30 & $(46,9)$ & 64 \\
\hline $\begin{array}{l}\text { Substanzen des } \\
\text { Verdauungstraktes }\end{array}$ & 59 & 27 & (46) & 89 & $(73,6)$ & 121 \\
\hline Vitamine & 46 & 16 & (35) & 3 & $(9,1)$ & 33 \\
\hline Hormone & 43 & 20 & (46) & 18 & $(43,9)$ & 41 \\
\hline Mineralien & 19 & 5 & (26) & 22 & $(61,2)$ & 36 \\
\hline Antibiotika & 11 & 5 & (45) & 55 & $(87,3)$ & 63 \\
\hline GESAMT & 1331 & 482 & (36) & 711 & $(45,5)$ & 1562 \\
\hline
\end{tabular}


Tabelle 8: Abgesetzte Medikamente

\begin{tabular}{|c|c|c|c|c|c|c|c|}
\hline \multirow{2}{*}{\multicolumn{8}{|c|}{$\begin{array}{ccc}\text { ABGESETZTE MEDIKAMENTE IM KRANKENHAUS } & 2 & 3 \\
1 & 2 & 3\end{array}$}} \\
\hline & & & & & & & \\
\hline $\begin{array}{l}\text { Medikamenten- } \\
\text { gruppen* }\end{array}$ & $\begin{array}{c}\text { abge- } \\
\text { setzt } \\
\mathrm{N}\end{array}$ & $\%$ & $\begin{array}{c}\text { abge- } \\
\text { setzt } \\
\text { N }\end{array}$ & $\%$ & $\begin{array}{c}\text { abge- } \\
\text { setzt } \\
\mathbf{N}\end{array}$ & $\%$ & Summe \\
\hline Antihypertensiva & 53 & (41) & 17 & (13) & 58 & (46) & 128 \\
\hline Substanzen des ZNS & 14 & (36) & 8 & (21) & 17 & (43) & 39 \\
\hline $\begin{array}{l}\text { Substanzen des } \\
\text { Verdauungstraktes }\end{array}$ & 8 & (30) & 6 & (22) & 13 & (48) & 27 \\
\hline $\begin{array}{l}\text { Substanzen des } \\
\text { Respirationstraktes }\end{array}$ & 9 & (31) & 18 & (62) & 2 & (7) & 29 \\
\hline $\begin{array}{l}\text { Kardiovaskuläre } \\
\text { Substanzen }\end{array}$ & 47 & (44) & 22 & (21) & 37 & (35) & 106 \\
\hline Antikoagulanzien & 12 & (28) & 9 & (21) & 22 & (50) & 43 \\
\hline Antibiotika & 2 & (40) & 1 & (20) & 2 & (40) & 5 \\
\hline Antirheumatika & 11 & (41) & 4 & (15) & 12 & (44) & 27 \\
\hline Mineralien & 1 & (20) & 0 & (0) & 4 & (80) & 5 \\
\hline Hormone & 3 & (15) & 5 & (25) & 12 & (60) & 20 \\
\hline Antidiabetika & 13 & (35) & 6 & (16) & 18 & (49) & 37 \\
\hline Vitamine & 8 & (50) & 5 & (31) & 3 & (19) & 16 \\
\hline GESAMT & 181 & $(38 \%)$ & 101 & $(21 \%)$ & 200 & $(41 \%)$ & 482 \\
\hline
\end{tabular}

Tabelle7-8: * laut ATC-Code 1998; Mineralien und Vitamine aus der Gruppe „Substanzen des Verdauungstraktes“" wurden zur besseren Übersicht extra gruppiert 
Bei den Substanzen des ZNS, den Substanzen des Verdauungstraktes und den Hormonen wurden prozentual die meisten Medikamente abgesetzt, wobei letztendlich in vielen dieser Stoffgruppen die Anzahl der Neuverordnungen höher war als der Ausgangswert der Praxismedikation (Tabelle 7 u. 8).

Die Veränderungen der Einweisungsmedikation hinsichtlich Generika bzw. Orginalpräparate sind in Tabelle 9 dargestellt. Hierbei wird deutlich, daß bei über der Hälfte aller Umstellungen im Krankenhaus Generika durch Orginalpräparate ersetzt wurden. Der Wechsel von Orginalpräparaten auf Generika wurde lediglich in 14 Fällen vollzogen. Von der Einweisungsmedikation wurden 46 Orginalpräparate abgesetzt und durch Orginalpräparate anderer Substanzen ersetzt. Insgesamt wurden $44,5 \%$ der hausärztlich verordneten Generika durch den Krankenhausarzt abgesetzt.

Vergleicht man die Veränderungen in den einzelnen Krankenhäusern, sind keine nennenswerten Unterschiede erkennbar.

Tabelle 9: Umstellung der Einweisungsmedikation im Krankenhaus auf Generika oder Orginalpräparate

\begin{tabular}{lccc|cr}
\hline Umstellung der & \multicolumn{7}{c|}{ Krankenhaus } & \multicolumn{2}{c}{} \\
Einweisungsmedikation & $\mathbf{1}$ & $\mathbf{2}$ & $\mathbf{3}$ & Summe & (\%) \\
\hline Generika zu Orginalpräparat & 14 & 10 & 14 & 38 & $(69)$ \\
Generika zu Generika & - & 1 & 2 & 3 & $(5)$ \\
Orginalpräparat zu Generika & 5 & 4 & 5 & 14 & $(26)$ \\
\hline GESAMT & 19 & $\mathbf{1 5}$ & $\mathbf{2 1}$ & 55 & $\mathbf{1 0 0 \%}$ \\
\hline
\end{tabular}




\subsection{Arzneimittel-Neuverordnungen im Krankenhaus}

Im Vergleich zur Hausarztmedikation wurden in den 3 Krankenhäusern insgesamt 711 Arzneimittel neu verschrieben (Tabelle 10). Die Antihypertensiva (25,7\%) wurden auch in den Krankenhäusern am häufigsten verordnet, gefolgt von Substanzen des ZNS $(13,2 \%)$ und Substanzen des Verdauungstraktes (12,5\%). Die geringste Anzahl an Neuverordnungen hatte Krankenhaus 2. 
Tabelle 10: Arzneimittel- Neuverordnungen

KRANKENHAUS

(NEUVERORDNUNGEN)

1

2

3

\begin{tabular}{|c|c|c|c|c|c|c|c|}
\hline $\begin{array}{l}\text { Medikamenten- } \\
\text { gruppen }\end{array}$ & $\mathbf{N}$ & (RANG) & $\mathbf{N}$ & (RANG) & $\mathbf{N}$ & (RANG) & GESAMT \\
\hline Antihypertensiva & 81 & (1) & 52 & (1) & 50 & (1) & 183 \\
\hline Substanzen des ZNS & 33 & (2) & 25 & (4) & 36 & (3) & 94 \\
\hline $\begin{array}{l}\text { Substanzen des Ver- } \\
\text { dauungstraktes }\end{array}$ & 27 & (4) & 27 & (2) & 35 & (4) & 89 \\
\hline $\begin{array}{l}\text { Substanzen des } \\
\text { Respirationstraktes }\end{array}$ & 24 & (5) & 27 & (2) & 37 & (2) & 88 \\
\hline $\begin{array}{l}\text { Kardiovaskuläre } \\
\text { Substanzen }\end{array}$ & 29 & (3) & 11 & (7) & 22 & (5) & 62 \\
\hline Antikoagulanzien & 24 & (5) & 11 & (7) & 20 & (7) & 55 \\
\hline Antibiotika & 16 & (7) & 18 & (5) & 21 & (6) & 55 \\
\hline Antirheumatika & 9 & (8) & 11 & (7) & 10 & (8) & 30 \\
\hline Mineralien & 2 & (11) & 13 & (6) & 7 & (9) & 22 \\
\hline Hormone & 5 & (10) & 6 & (10) & 7 & (9) & 18 \\
\hline Antidiabetika & 6 & (9) & 3 & (11) & 3 & (11) & 12 \\
\hline Vitamine & 1 & (12) & 2 & (12) & 0 & (12) & 3 \\
\hline GESAMT & 257 & & 206 & & 248 & & 711 \\
\hline
\end{tabular}


In den Krankenhäusern betrug der Anteil der Orginalpräparate an den Neuverordnungen 79\%. Betrachtet man alle im Krankenhaus verabfolgten Medikamente (die Übernahme der Hausarztmedikamente und die Krankenhausneuverordnungen) resultiert ein Generikaanteil von 20,2\%. In den Hausarztpraxen sind durchschnittlich $26 \%$ Generika verordnet worden (Tabelle 11).

Tabelle 11: Orginalpräparate und Generika der Krankenhäuser (Neuverordnungen) und der Hausarztpraxen

\begin{tabular}{|c|c|c|c|c|}
\hline \multirow[t]{2}{*}{ KRANKENHAUS } & \multicolumn{2}{|c|}{ GENERIKA } & \multicolumn{2}{|c|}{ ORGINALPRÄPARAT } \\
\hline & $\mathbf{N}$ & $(\%)$ & $\mathbf{N}$ & (\%) \\
\hline 1 & 49 & $(19,5)$ & 205 & $(80,5)$ \\
\hline 2 & 45 & $(22,1)$ & 161 & $(77,9)$ \\
\hline 3 & 54 & $(21,7)$ & 197 & $(78,3)$ \\
\hline Gesamt & 148 & $(21 \%)$ & 563 & $(79 \%)$ \\
\hline Hausarztpraxen & 344 & $(26 \%)$ & 987 & $(74 \%)$ \\
\hline
\end{tabular}

\subsection{Patientenaufklärung}

Bei insgesamt 16 der 300 in die Studie eingeschlossenen Patienten war eine Befragung auf Grund fortgeschrittener Demenz, gesundheitlich starker Beeinträchtigung oder aus persönlichen Gründen (Verweigerung) nicht möglich.

Im Krankenhaus 1 konnten sich ca. $85 \%$ der Patienten an eine Aufklärung über etwaige Arzneimittel erinnern. In den Krankenhäusern 2 und 3 waren es etwas weniger Patienten (Tabelle 12). 
Tabelle 12: Aufklärung der Patienten über medikamentöse Veränderungen in den Krankenhäusern

\section{Aufklärung der Patienten}

\section{KRANKENHAUS}

1

2

3

$\begin{array}{llllll}\mathbf{N} & (\%) & \mathbf{N} & (\%) & \mathbf{N} & (\%)\end{array}$

$J A$

$\begin{array}{llllll}80 & 85,1 & 78 & 83,9 & 76 & 78,3\end{array}$

NEIN

$14 \quad 14,9 \quad 15 \quad 16,1 \quad 21 \quad 21,5$

Betrachtet man die Aufklärung in Abhängigkeit vom Alter (Abbildung 1) nimmt die Häufigkeit der Aufklärung mit zunehmenden Alter ab.

Abbildung 1: Aufklärung der Patienten über medikamentöse Veränderungen

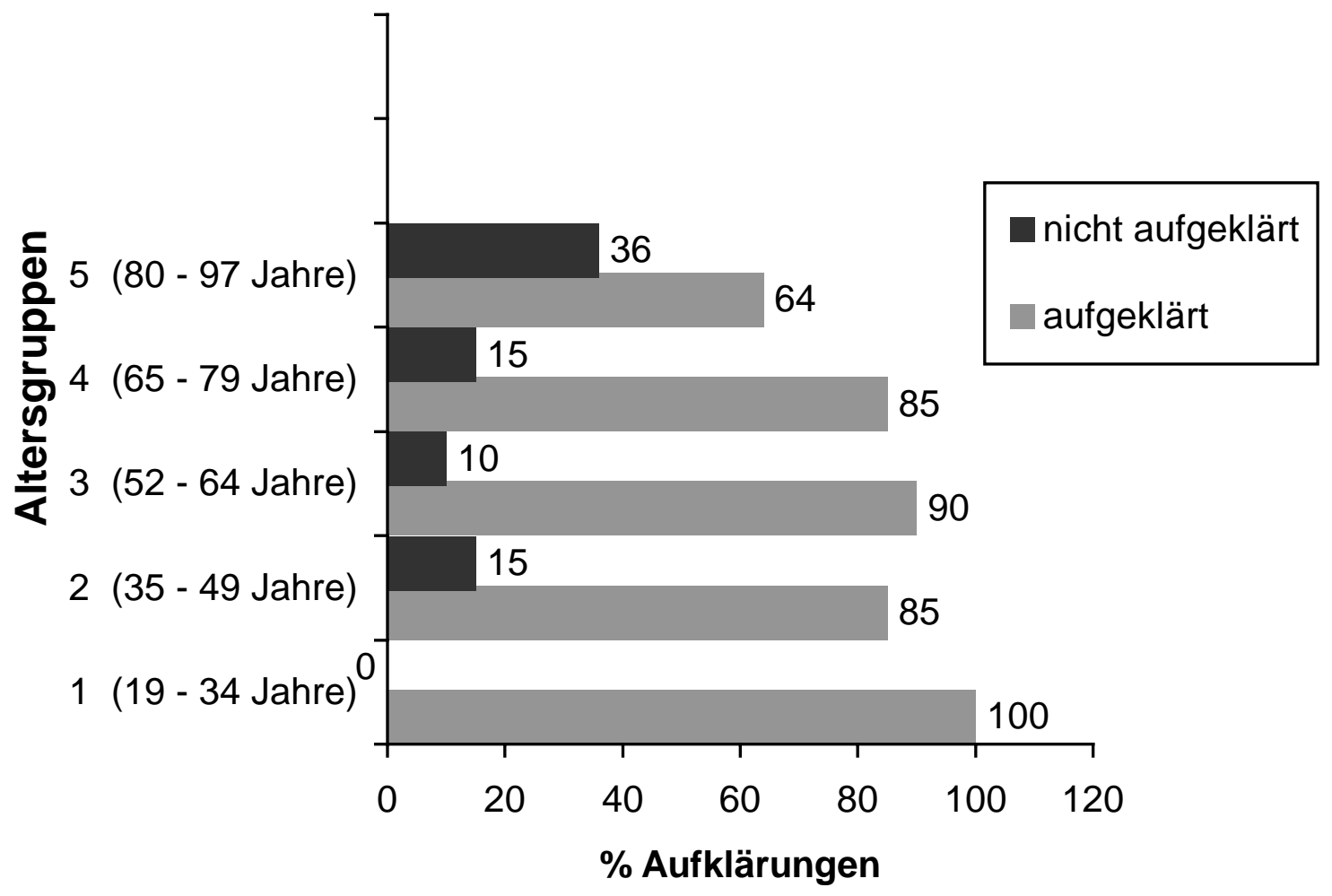


In $77 \%$ der Fälle wurden die Patienten durch den Arzt aufgeklärt. Bei den anderen Patienten informierten Pflegepersonal oder sogar Angehörige über Veränderungen der Medikation.

Das Aufklärungsgespräch ist von $77 \%$ der Patienten als wichtig eingestuft worden. In der Gruppe der 80-97 jährigen Patienten wurde das Aufklärungsgespräch am wenigsten für wichtig erachtet.

In den meisten Fällen fand die Aufklärung der Patienten im Krankenzimmer bei der täglichen Visite statt.

Tabelle 13: Bedeutung der Aufklärung für die Altersgruppen

\begin{tabular}{lcccc} 
Altersgruppe & \multicolumn{5}{c}{ Aufklärung wichtig? } \\
& ja & $(\%)$ & nein & $(\%)$ \\
\hline $\mathbf{1}(19-34$ Jahre $)$ & 4 & $(80)$ & 1 & $(20)$ \\
$\mathbf{2}(35-49$ Jahre $)$ & 11 & $(85)$ & 2 & $(15)$ \\
$\mathbf{3}(52-64$ Jahre $)$ & 41 & $(85)$ & 7 & $(15)$ \\
$\mathbf{4}(65-79$ Jahre $)$ & 133 & $(79)$ & 35 & $(21)$ \\
$\mathbf{5}$ (80 - 97 Jahre) & 29 & $(58)$ & 21 & $(42)$ \\
\hline GESAMT & $\mathbf{2 1 8}$ & $\mathbf{( 7 7 \% )}$ & $\mathbf{6 6}$ & $(\mathbf{2 3} \%)$ \\
\hline
\end{tabular}

Die 284 Patienten wurden nach dem Inhalt des Aufklärungsgespräches befragt. (Tabelle 14). Jeder vierte Patienten war über die Wirkung des neuen Arzneimittels und den Grund für diese Veränderung informiert worden. Lediglich $16 \%$ wurden über die Dosis des neuen Medikaments in Kenntnis gesetzt. Eine vollständige Aufklärung der Patienten über Medikamentenname, Wirkung, Dosis, Einnahmevorschrift und Änderungsgrund erfolgte nur in etwa 10\% der Fälle. 
Tabelle14: Inhalt der Aufklärungsgespräche

\begin{tabular}{|c|c|c|c|c|c|c|c|c|c|c|}
\hline \multicolumn{11}{|c|}{ A U F K L ÄR UNGSINHALT } \\
\hline ALTERSGRUPPE & \begin{tabular}{|l} 
Name / \\
Wirkung
\end{tabular} & $\begin{array}{l}\text { Name/ } \\
\text { Wirkung/ } \\
\text { Dosis }\end{array}$ & $\begin{array}{l}\text { Name/ } \\
\text { Wirkung/ } \\
\text { Dosis/ } \\
\text { Vorschrift }\end{array}$ & \begin{tabular}{|l} 
Name/ \\
Wirkung/ \\
Dosis/ \\
Vorschrift/ \\
Änderung
\end{tabular} & \begin{tabular}{|l} 
Name/ \\
Vorschrift/ \\
Änderung
\end{tabular} & \begin{tabular}{|l|} 
Name/ \\
Änderun- \\
gen
\end{tabular} & \begin{tabular}{|l} 
Wirkung/ \\
Änderung
\end{tabular} & \begin{tabular}{|l} 
Wirkung/ \\
Einnahme/ \\
Änderungen
\end{tabular} & Sonstiges & $\begin{array}{c}\text { Gesamt } \\
\text { N }\end{array}$ \\
\hline 1 (19 - 34 Jahre) & - & - & - & 1 & 1 & - & 1 & - & 2 & 5 \\
\hline 2 (35 - 49 Jahre) & 3 & - & 1 & 2 & - & - & 1 & 1 & 3 & 11 \\
\hline 3 (52 - 64 Jahre) & 9 & 3 & 3 & 8 & 8 & - & 7 & 2 & 4 & 44 \\
\hline 4 (65 - 79 Jahre) & 20 & 5 & 4 & 11 & 28 & 7 & 38 & 10 & 18 & 141 \\
\hline $\begin{array}{ll}5 & \text { (80 - } 97 \text { Jahre) }\end{array}$ & 2 & - & - & 1 & 4 & 2 & 11 & 5 & 8 & 33 \\
\hline GESAMT & 34 & 8 & 8 & 23 & 41 & 9 & 58 & 18 & 35 & \\
\hline
\end{tabular}


Um zu erfahren, was sich die Patienten von der Aufklärung merken konnten, wurden spezielle Fragen (Präparatename, Dosis usw.) über die neu verordneten Medikamente gestellt (Tabelle 15). Auffallend war die geringe Detailkenntnis. 120 Patienten wurde beispielsweise der Medikamentenname mitgeteilt, aber nur 37\% der Patienten waren in der Lage, diesen zu benennen. Über die Dosis konnten 9 Befragte eine Auskunft geben. 60\% der Patienten waren in der Lage den Grund für die Verschreibung der Medikamente zu benennen. Im Vergleich der Kliniken war im Krankenhaus 3 eine generell geringere Detailkenntnis festzustellen.

Tabelle 15 zeigt den Wissensstand in den einzelnen Altersgruppen. Auffallend war die rückläufige Kenntnis über die neu verordneten Medikamente, die eindeutig mit dem steigendem Patientenalter korrelierte.

Tabelle 15: Wissen der Patienten über Neuverordnungen

\begin{tabular}{|c|c|c|c|c|c|c|c|c|}
\hline \multirow[b]{3}{*}{ Wissen der Patienten } & \multicolumn{8}{|c|}{ KRANKENHAUS } \\
\hline & \multicolumn{2}{|c|}{1} & \multicolumn{2}{|c|}{2} & \multicolumn{2}{|c|}{3} & \multicolumn{2}{|c|}{ Alle } \\
\hline & ja & $(\%)$ & ja & $(\%)$ & ja & $(\%)$ & ja & (\%) \\
\hline $\begin{array}{l}\text { Name des Medikaments } \\
\text { bekannt? }\end{array}$ & 16 & (21) & 19 & (26) & 11 & (14) & 46 & $(16,2)$ \\
\hline $\begin{array}{l}\text { Dosis des Medikaments } \\
\text { bekannt? }\end{array}$ & 3 & (4) & 4 & $(4)$ & 2 & (3) & 9 & $(3,2)$ \\
\hline $\begin{array}{l}\text { Einnahmevorschrift des Medi- } \\
\text { kaments bekannt? }\end{array}$ & 27 & (35) & 24 & (33) & 19 & $(26)$ & 70 & $(24,6)$ \\
\hline $\begin{array}{l}\text { Grund der Verordnung } \\
\text { bekannt? }\end{array}$ & 52 & (68) & 50 & (70) & 37 & $(50)$ & 139 & $(48,9)$ \\
\hline
\end{tabular}


Tabelle 16: Wissen der Altersgruppen über Neuverordnungen

\begin{tabular}{|c|c|c|c|c|c|c|c|c|}
\hline \multirow[b]{3}{*}{ Altersgruppe } & \multicolumn{8}{|c|}{ AUFKLÄRUNG ÜBER } \\
\hline & \multicolumn{2}{|c|}{ Handelsname } & \multicolumn{2}{|c|}{ Dosis } & \multicolumn{2}{|c|}{ Einnahme } & \multicolumn{2}{|c|}{$\begin{array}{c}\text { Grund der } \\
\text { Verschreibung }\end{array}$} \\
\hline & ja & (\%) & ja & $(\%)$ & ja & $(\%)$ & ja & $(\%)$ \\
\hline 1 (19 - 34 Jahre) & 2 & $(40)$ & 2 & $(40)$ & 4 & (80) & 3 & $(60)$ \\
\hline 2 (35 - 49 Jahre) & 4 & (36) & 1 & $(0,9)$ & 5 & $(45)$ & 8 & $(73)$ \\
\hline 3 (52 - 64 Jahre) & 15 & (35) & 2 & $(0,5)$ & 20 & $(46)$ & 33 & $(77)$ \\
\hline 4 (65 - 79 Jahre) & 22 & (15) & 4 & $(0,3)$ & 37 & (26) & 86 & $(60)$ \\
\hline 5 (80 - 97 Jahre) & 3 & $(0,9)$ & 0 & (0) & 4 & (12) & 9 & $(28)$ \\
\hline GESAMT & 46 & $(20 \%)$ & 9 & $(3,8 \%)$ & 70 & $(30 \%)$ & 139 & $(59 \%)$ \\
\hline
\end{tabular}




\section{Diskussion}

Schwerpunkt der vorliegenden Arbeit war die Frage, was mit der hausärztlichen Medikation nach Krankenhauseinweisung geschieht. Es wurde untersucht, inwieweit Medikamente durch den Klinikarzt übernommen, abgesetzt oder ersetzt werden. Parallel sollte geklärt werden, wie gut sich Patienten über Veränderungen ihrer Medikation informiert fühlen und ob sie ihre neuen Medikamente kennen.

Die Hälfte der hausärztlich verordneten Medikamenten wurden verändert, 36\% wurden abgesetzt und bei 15\% der Arzneimittel änderte sich die Dosis bzw. der Hersteller. Neu verordnet wurden 711 Medikamente. Der Anteil von Generika sank von 25,5\% (Praxis) auf $20,2 \%$ in den Krankenhäusern. 82\% der interviewten Patienten wurden über die Medikationsänderungen aufgeklärt.

\section{1 Methode}

Im Gegensatz zu vorangegangenen Arbeiten aus Universitätskliniken (Harder et al. 1991, Himmel et al. 1996b) wurde die Studie in drei Akutkrankenhäusern einer Region durchgeführt. Die hier vorgelegten Daten sind daher nicht durch die Besonderheit „wissenschaftlicher“ Medizin (Göpel 1986) geprägt. Die Auswahl mehrerer Krankenhäuser gleicher Kategorie innerhalb einer Region ermöglicht zudem eine Verallgemeinerung der Ergebnisse. Im Gegensatz zur Arbeit von Himmel et al. (1996a), der eine ähnliche Thematik aus Sicht einer Praxis untersuchte, schließt ein krankenhausbezogener Ansatz ein breites Spektrum von Hausarztpraxen und deren Verordnungen ein und ist auch deshalb eher verallgemeinerungsfähig.

Die Untersuchungen fanden auf den internistischen Stationen der einzelnen Krankenhäuser statt, da Hausärzte ihre Patienten überwiegend in diese Abteilungen einweisen und Internisten unter den im Krankenhaus tätigen Ärzten am häufigsten Medikamente umstellen (Himmel et al. 1996b). 
Die Datenerhebung der bis zum Aufnahmezeitpunkt verordneten Hausarztmedikamente sowie der Diagnosen erfolgte anhand des Einweisungsscheines und der Krankenhausanamnese. Die lückenlose Dokumentation der Vorerkrankungen und Medikation waren von der Sorgfalt des einweisenden bzw. aufnehmenden Arztes und vom Erinnerungsvermögen der Patienten abhängig. Da $76 \%$ der befragten Patienten bereits 65 Jahre und älter waren, muß mit einer erhöhten Anzahl von Fehlern bei der Angabe der Medikation gerechnet werden (Mahdy und Seymour 1990).

Die Krankenhausmedikamente zur Therapie des Diabetes mellitus und der Thromboseprophylaxe wurden teilweise in gesonderten Akten geführt. Diese standen nicht immer zur Verfügung, so daß beide Medikamentengruppen teilweise unvollständig dokumentiert sind.

Die Befragung zur Qualität der Aufklärung über medikamentöse Veränderungen wurde bei allen Patienten der Studie im Anschluß an das Aktenstudium mit Hilfe des Fragebogens (Anhang, S. 55-57) von einer Person durchgeführt. Die Beeinflussung der Ergebnisse durch unterschiedliche "Befragungsstile“ ist somit weitgehend ausgeschlossen.

Nur 16 der 300 ausgewählten Patienten konnten nicht in die Studie einbezogen werden. Ein Patient lehnte das Interview ab, 8 Personen konnten auf Grund ihrer fortgeschrittenen cerebrovaskulären Insuffizienz nicht befragt werden. Bei 7 Patienten blieb die Medikation unverändert, so daß ein Interview nicht sinnvoll erschien. Somit lag die Response-Rate bei nahezu 100\%.

Die Fragen über die zusätzliche Einnahme hausärztlich verschriebener Medikamente und über Selbstmedikation im Krankenhaus (Anhang, S. 57, Fragen 9 -11) wurden überwiegend mit nein beantwortet. Da die Selbstmedikation auf dem deutschen Arzneimittelmarkt bei $41 \%$ aller Medikamente liegt (Bundesfachverband der Arzneimittel-Hersteller 1999), dürften die Antworten nur bedingt zutreffen und häufig im Sinne „sozialer Erwünschtheit“ geprägt sein. 


\subsection{Ergebnisse}

Basierend auf den Studienergebnissen sollen nun die Hypothesen diskutiert werden.

\section{Hypothese}

Über die Hälfte der hausärztlich verordneten Medikamente wird in den Krankenhäusern verändert.

Bis zum Zeitpunkt der stationären Aufnahme erhielten die Patienten durchschnittlich 4,4 Medikamente vom Hausarzt verordnet. Vergleichbar ist die Zahl mit der Arbeit von Beers et al (1989). In dieser Studie erhielten die Patienten im Durchschnitt 4,5 Medikamente, das Alter der Patienten lag bei ca. 75 Jahre.

Die Internisten übernahmen nur $48.6 \%$ der hausärztlich verordneten Medikamente; über $36 \%$ der Arzneimittel setzten sie ab. Bei den restlichen Medikamenten wurden Dosierungen und/oder Hersteller verändert. Diese Zahlen bestätigen die Ergebnisse vorangegangener Arbeiten. Zwischen 30\% und 40\% der vom Hausarzt verordneten Pharmaka setzten Klinikärzte in den Studien von Himmel et al. (1996b) und Beers et al. (1989) ab.

Im Krankenhaus bekamen die 300 Patienten 1562 Medikamente und somit durchschnittlich 0,8 Medikamente mehr als bei der Einweisung. Eine Zunahme der verordneten Medikamente nach Krankenhauseinweisung berichten fast alle Untersuchungen. So stieg die Zahl verordneter Arzneimittel in einer Arbeit von Beers et al. (1989) von durchschnittlich 4,5 bei Aufnahme auf 4,8 Präparate bei Entlassung. Andere Studien fanden sogar eine Verdopplung der Medikamente im Krankenhaus (Lucena Gonzalez et al. 1995, Gosney und Tallis 1984). Bei Kruse et al. (1990) sank hingegen die Anzahl der Medikamente nach stationärer Aufnahme um ca. ein Drittel. Regionale Gegebenheiten und andere Faktoren könnten Einfluß auf die Ergebnisse haben. 
Von den 711 im Krankenhaus neu verordneten Medikamenten entfielen die meisten auf Stoffgruppen, die auch am häufigsten abgesetzt wurden. Ein Großteil der neuen Medikamente diente also dem Austausch der Stoffklassen innerhalb der einzelnen Arzneimittelgruppen.

Die Gründe der Krankenhausärzte für die Veränderungen der Hausarztmedikation sind vermutlich vielfältig. Ausschlaggebend ist zunächst die Fachrichtung, im vorliegenden Fall die Beschränkung auf internistische Stationen: Vor allem Internisten stellen vermehrt Einweisungsmedikamente um (Himmel et al. 1996a).

Die Angst vor toxischen Nebenwirkungen und Wechselwirkungen der Verordnungen führt wahrscheinlich ebenfalls zu Veränderungen der Hausarztmedikation. Beers et al. (1989) gehen davon aus, daß 75\% der ehemals verordneten und im Krankenhaus abgesetzten Medikamente wirkungslos oder unnötig waren. Jedoch sollte erwähnt werden, daß auch auf internistischen Stationen Entlassungsmedikamente mit erhöhtem Potential für gefährliche Wechselwirkungen verschrieben werden (Bonetti et al. 2000).

Die „Rote Liste 1999“ enthält 9493 unterschiedliche Präparate. Deren Neben- und Wechselwirkungen wird der praktisch tätige Arzt kaum zur Gänze kennen.

Tomson et al. nehmen an, daß Allgemeinärzte über ungefähr 50 Medikamente detailliertes Wissen haben und mit weiteren 150 vertraut sind (Tomson et al.1994).

Auch die Verfügbarkeit in der Klinikapotheke entscheidet mit darüber, ob ein ambulant verordnetes Medikament im Krankenhaus weitergeführt oder abgesetzt bzw. durch ein ähnliches Medikament fortgeführt wird (Harder et al. 1991). 


\section{Hypothese}

Antihypertensiva und kardiovaskuläre Substanzen sind in den Krankenhäusern am häufigsten von Änderungen betroffen.

In der vorliegenden Studie verordneten sowohl die Allgemeinärzte als auch die Krankenhausärzte in der weiterführenden Behandlung am häufigsten Antihypertensiva und kardiovaskuläre Substanzen. Bedingt ist der Einsatz dieser Substanzgruppen in erster Linie durch das hohe Durchschnittsalter der untersuchten Patienten. In der Arbeit von Rokstad et al. (1997) erhielten ungefähr ein Drittel der untersuchten 7770 Patienten im Alter von 60 bis über 80 Jahren kardiovaskuläre Substanzen. Ebenso war ein Großteil der stationären Aufenthalte der von Kruse et al. (1990) untersuchten Patienten im Alter von 60-98 Jahren durch kardiovaskuläre Erkrankungen bedingt. Von Bedeutung für dieses Ergebnis ist auch die ausschließliche Konzentration der Studie auf Allgemeinarztpraxen und internistische Stationen: Der Anteil der von Allgemeinärzten und Internisten verordneten Antihypertensiva und kardiovaskulären Substanzen an deren Gesamtverordnung liegt besonders hoch (Schröder und Selke 2000 ).

In den Krankenhäusern setzten die Internisten 30\% bzw. knapp 40\% der Antihypertensiva und kardiovaskulären Substanzen ab und verordneten diese in ähnlichem Umfang neu. Häufiger waren Antibiotika und Substanzen des ZNS- und Verdauungstraktes von Veränderungen betroffen. Medikamente dieser Arzneimittelgruppen wurden im Krankenhaus besonders oft neu verordnet, vergleichsweise seltener zuvor abgesetzt, so daß man in diesen Fällen eher von einem Austausch der Substanzklassen sprechen muß.

In vergleichbaren Studien wird die Veränderung der Hausarztmedikamente in den Krankenhäusern bei bestimmten Substanzgruppen besonders deutlich. Die Angaben über die Medikamentengruppen mit dem höchsten Änderungspotential sind allerdings sehr unterschiedlich, so daß der Vergleich mit den vorliegenden Ergebnissen ein nur bedingt kohärentes Bild ergibt. 
Beers et al. (1989) registrierten eine erhöhte Verordnung von Antibiotika, Analgetika und Kardiaka. Bei Lucena Gonzales et al. (1995) resultiert eine Verdopplung der Medikamente nach Krankenhauseinweisung aus der verstärkten Verschreibung von Antibiotika, Digestiva und Hämatologika. In der Studie von Kruse et al. (1990) setzten die Ärzte u.a. häufig Antihypo- und Antihypertonika, Diuretika sowie Benzodiazepine ab und verordneten vor allem Antidepressiva, Thyreostatika, Antikoagolantien, H2-Blocker und Neuroleptika.

Gründe für die Differenzen zwischen den einzelnen Studien könnten in der Zusammensetzung der untersuchten Stationen liegen. Die unterschiedlichen Krankheitsbilder lassen kaum spezifische Vergleiche bei den Änderungen innerhalb einzelner Medikamentengruppen zu. Das „individuelle“ Verordnungsprofil der einzelnen Krankenhäuser dürfte gleichfalls eine Rolle spielen (Himmel et al. 1996b ).

\section{Hypothese}

Krankenhausärzte verschreiben deutlich weniger Generika als Hausärzte

Der Anteil der Generika an der Gesamtmedikation der Patienten betrug bei Aufnahme ins Krankenhaus 25,5\%. Im Krankenhaus verringerte sich der Anteil der Generika um ca. $5 \%$ auf $20,2 \%$.

Niedergelassene Ärzte sind seit der Gesundheitsreform gezwungen, wirtschaftlicher zu arbeiten, und setzen daher verstärkt kostengünstige Generika ein. Dies gilt besonders für Allgemeinärzte, deren Therapie in erheblichen Umfang auf Medikamenten beruht. 1999 lag der Anteil hausärztlich verordneter Generika am Gesamtverordungsvolumen bei 51\% (Schröder u. Selke 2000). Im Vergleich mit den Ergebnissen zur ähnlich angelegten Studie von Himmel et al. (1998) fällt auf, daß Allgemeinärzte Generika immer konsequenter nutzen. So verdoppelte sich deren Einsatz innerhalb von 5 Jahren (Daten der Studie von 1994) von 13,5 auf nunmehr 25,5\%. Gefördert wird dieses Verhalten durch das ständig größer werdende 
Angebot, aber auch die verbesserte Aufklärung des Arztes über Kosten und Nutzen der Generika (Beilby und Silagy 1997).

Dem mittlerweile auch auf Klinikärzten lastende wirtschaftliche Druck wird offensichtlich bisher nicht so oft wie im primärärztlichen Bereich durch einen Rückgriff auf Generika begegnet. Auch Spezialisten verordnen offensichtlich seltener Generika. Nach einer Studie von Bijl et al. (1998) änderten sie bei 43,6\% der Patienten die Medikation so erheblich, daß die Kosten um über 23\% anstiegen. Dies hat zum Teil auch damit zu tun, daß bei vielen Präparaten von Spezialisten keine Generika zur Verfügung stehen. Die Tendenz junger Ärzte, die Verordnungsgewohnheiten älterer Kollegen zu übernehmen (Shulkin et al. 1992), könnte ein weiterer Grund für die vermehrte Verschreibung von teureren Originalpräparaten in Krankenhäusern sein.

Die oft kostspieligen Krankenhausverordnungen haben eine enorme Wirkung auf das Arzneimittelbudget der Allgemeinärzte. Diese sind dadurch nicht immer in der Lage, ihre Kosten selbst zu kontrollieren (Stevenson et al. 2000). Durch die ungleiche Gewichtung des ökonomische Aspektes wird das Verhältnis zwischen Praxis und Krankenhaus oft nachhaltig gestört (Jones und Rawlins 1992). Zumal sich der Kliniker laut Petersen (2000) darüber im Klaren sein muß, daß seine Patienten nach Entlassung gegebenenfalls wieder auf ein Generikum umgestellt werden. Meist fällt es dem Allgemeinarzt jedoch schwer, die durch das Krankenhaus verschriebenen Medikamente wieder zu ändern, da er damit seine Beziehung zum Patienten aber auch zum Klinikarzt gefährden könnte (Stevenson et al 2000).

Die vorliegenden Ergebnisse deuten darauf hin, daß die Kluft zwischen Allgemeinärzten und Krankenhaus-Internisten beim Einsatz von Generika kleiner wird. Klinikärzte setzen verstärkt kostengünstigere Medikamente ein, was den Allgemeinärzten die weiterführende Behandlung erleichtert. Auch Marshall (1999) weist darauf hin, daß trotz bestehender Differenzen zwischen beiden Bereichen eine verstärkt positive Einstellung zur Zusammenarbeit besteht. 


\section{Hypothese}

Kommunale Krankenhäuser ändern die Hausarztmedikation seltener als Universitätskliniken

In dieser Studie zeigten sich gewisse Unterschiede bezüglich des Verordnungsprofils zwischen den internistischen Stationen von drei Krankenhäusern gleicher Größenordnung: Während die Internisten in den Krankenhäusern 1 und 3 ca. 55\% der Einweisungsmedikamente umstellten, wurden im Krankenhaus 2 nur 42\% verändert. Das Verordnungsprofil könnte von institutionellen Besonderheiten beeinflußt sein, die hier nicht weiter untersucht wurden.

Bisher ging man davon aus, daß die Größe des Krankenhauses einen erheblichen Einfluß auf den Umfang der Veränderungen hat. Gerade in Universitätskliniken mit einer großen Anzahl an Spezialisten und Wissenschaftlern ist ein kritischer Umgang mit der Hausarztmedikation anzunehmen, zumal die Therapie in großen Kliniken laut Göpel (1986) „wissenschaftsorientiert“ ist und oft von der Klinikleitung vorgegeben wird. Himmel et al. (1996b) konnten keine signifikanten Verordnungsunterschiede zwischen Universitätsklinik und Krankenhäusern, die unserer Kategorie entsprechen, feststellen. Allerdings lag die Anzahl der abgesetzten hausärztlich verordneten Medikamente in beiden Typen von Kliniken (Universität und Kreiskrankenhaus) mit ca. $65 \%$ fast doppelt so hoch wie in dieser Arbeit. Die genauere Aufklärung möglicher Unterschiede zwischen kommunalen Krankenhäusern und Universitätskliniken bedarf weiterer Untersuchungen. 


\section{Hypothese}

Patienten wissen nach der Aufklärung über Veränderungen ihrer Medikation nicht entscheidend mehr als vorher.

Nach Krankenhauseinweisung wurden über die Hälfte der hausärztlich verordneten Arzneimittel umgestellt. Auf Grund dieser massiven Veränderungen von Medikamenten, an deren Einnahme sich die Patienten oft schon über Jahre gewöhnt hatten, sollte man eine sorgfältige Aufklärung erwarten. Geschieht dies nicht, sind Einnahmefehler, vermehrte Nebenwirkungen und in der Folge eine geringere $\mathrm{Pa}$ tientencompliance sowie Belastung der Arzt-Patienten-Beziehung wahrscheinlich.

Eine angemessene Aufklärung der Patienten über Veränderungen ihrer Langzeitmedikation ist für die Compliance von großer Bedeutung (Cochrane et al. 1992). Offensichtlich wird in der täglichen Praxis der Patienteninformation jedoch nur geringe Aufmerksamkeit geschenkt. In der Studie von Shabbir et al. (1999) wurde z.B. nur die Hälfte der Patienten nach Krankenhauseinweisung über Veränderungen ihrer Medikation unterrichtet. Auch wenn Dowell et al. (1995) die Aufklärungspraktiken von Hausärzten als nicht zufriedenstellend kritisieren, scheint die Aufklärung in den Krankenhäusern noch ineffektiver zu sein. So ist die Patientencompliance vergleichsweise niedriger, wenn ein Medikament von einem Krankenhausarzt verschrieben wird (Spagnoli et al. 1989). Dieser Umstand könnte nach Meinung von Strobach et al. (2000) durch einen klinikinternen pharmazeutischen Beratungsdienst gebessert werden, da nach einem stationären Aufenthalt vor allem die Patienten die Verantwortung für die korrekte Medikamenteneinnahme tragen. Somit könnte eine gezielte Informationspolitik nach Beendigung der stationären Behandlung zu einer Steigerung der Patientencompliance und Therapieeffektivität führen. 
In der vorliegenden Arbeit konnte eine Aufklärungsrate von fast $80 \%$ ermittelt werden. Zwischen einzelnen Kliniken fanden sich keine nennenswerten Unterschiede.

Eine Erklärung für diese signifikante Abweichung von den Ergebnissen bisheriger Untersuchungen bleibt vorläufig spekulativ. Drei Gründe sind denkbar: (1) Der häufige Hinweis auf die Wichtigkeit der Patientenaufklärung veranlaßt mittlerweile Krankenhausärzte, die Mehrzahl der Patienten über den Wechsel von Medikamenten zu informieren; (2) in den hier ausgewählten Krankenhäusern müssen sich Ärzte stärker als in Universitätskliniken um die Patienten bemühen, was z.B. die Informationsqualität betrifft; (3) die hier gemessene Patientenaufklärung entspricht nicht den Kriterien anderer Studien, die möglicherweise höhere Anforderungen stellten und daher zu schlechteren Ergebnissen kommen. Diese dritte Vermutung - daß nämlich trotz Aufklärung die Kenntnisse der Patienten häufig mangelhaft bleiben - entspricht den Ergebnissen von Mahdy und Seymour (1990). Nur ca. $10 \%$ der Patienten waren hier in der Lage, eine vollständige Liste ihrer Medikamente zu nennen. Auch unsere Ergebnisse zeigen ein ähnliches Bild. Lediglich 20\% der aufgeklärten Patienten konnten den Namen ihres oder ihrer Medikamente nennen. An die korrekte Dosierung erinnerten sich nicht einmal 5\%. Allerdings konnten $63 \%$ den Verordnungsgrund angeben. Betrachtet man den Informationsgehalt der Aufklärungsgespräche dieser Studie, fällt auf, daß nur jeder vierte Patient über die Wirkung des neuen Medikaments und den Änderungsgrund unterrichtet wurde. Eine vollständige Information über Medikamentenname, Wirkung, Dosis, Einnahmevorschrift und Änderungsgrund erfolgte nur bei etwa $10 \%$ der befragten Patienten. Diese Ergebnisse beruhen wahrscheinlich auf der Gedächtnisleistung der Befragten, so daß die Zuverlässigkeit eingeschränkt sein kann. Ein Teil der Patienten könnte im Rahmen der Erwünschtheit geantwortet haben.

Ein weiterer, nicht zu unterschätzender Grund für schlechte Aufklärungsergebnisse ist die mangelnde Fähigkeit mancher Patienten, während der Konsultationen gezielt nach dem eigenen Gesundheitszustand bzw. dem Fortgang diagnostischer 
und therapeutischer Maßnahmen zu fragen (Barry et al. 2000). Aufgrund des geringen Wissens über die eigenen Medikamente dürfte es häufig zu Einnahmefehlern kommen (Shabbir et al. 1999).

Nach Kochen (1998) nehmen ca. die Hälfte aller Patienten ihre Medikamente nicht richtig, unregelmäßig oder überhaupt nicht ein.

\section{Hypothese}

Jüngere Patienten werden häufiger durch den Krankenhausarzt über Medikationsänderungen aufgeklärt.

Wichtig für das Verständnis der Patienten sind Art und Weise der Informationsvermittlung durch den behandelnden Arzt. Neben Kommunikationsqualität, Bildung, Intelligenzquotient und kognitiven Fähigkeiten hat das Alter des Patienten eine entscheidende Bedeutung auf die Informationsverarbeitung (Vollmann und Helmchen 1997).

$82,5 \%$ der Patienten dieser Studie wurden über Änderungen ihrer Medikation aufgeklärt. Bei genauer Betrachtung der Altersgruppen fällt im Alter zwischen 80 und 97 Jahren die Aufklärungsrate auf $64 \%$ ab. Hingegen lag sie bei den übrigen Altersgruppen zwischen $85 \%$ und $100 \%$. Dies könnte unter anderem am mangelnden Interesse der Patienten liegen, sich aufklären zu lassen. Schmeling-Kludas et al. (1989) machte mit steigendem Patientenalter ein sinkendes Informations- $u$. Aufklärungsbedürfnis der Patienten aus, wobei sie sich oft mit Erklärungen durch das Pflegepersonal zufrieden gaben. Immerhin $71 \%$ unserer Patienten hielten es für sehr wichtig, direkt durch den Arzt aufgeklärt zu werden.

Ein weiterer Grund für die mangelhafte Aufklärung vor allem älterer Patienten könnte in der schlechten Qualität der Arzt-Patientengespräche zu finden sein. 7080\% der Patienten fühlten sich durch Zeitmangel und Unverständlichkeit der ärzt- 
lichen Aufklärung gestört (Vollmann und Helmchen 1997). Ärzte beklagen ebenfalls, zu wenig Zeit für eine ordentliche Patientenaufklärung zur Verfügung zu haben (Shabbir et al. 1999).

Auch Preston et. al (1999) beschreiben Kommunikationsbarrieren zwischen Klinikarzt und Patient. Ausgelöst werden diese u.a. durch die vom Patienten empfundene „Höherstellung“ des Arztes. Vor allem ältere Menschen erstarren bei Arzt-Patientengesprächen häufig in Ehrfurcht. 


\section{Zusammenfassung}

Hintergrund: Vorwiegend aus Studien in Universitätskliniken bzw. aus Einzelpraxen ist bekannt, daß die Einweisung eines Patienten in ein Krankenhaus häufig die Änderung einer oft bereits seit langem bestehenden Medikation nach sich zieht. Ein Teil der Patienten wird nur unvollständig über diese Änderung aufgeklärt.

Ziel und Methode: Änderungen der hausärztlichen Medikation bei Einweisung und die Information der Patienten hierüber sollten auf internistischen Stationen von 3 Kreiskrankenhäusern Thüringens erhoben werden (chart review; standardisierte Interviews).

Ergebnisse: Die Medikamente von 300 Patienten (100 je Krankenhaus) vor und während des stationären Aufenthaltes wurden erhoben; 1 Patient verweigerte das anschließende Interview, 8 Patienten konnten aus gesundheitlichen Gründen nicht befragt werden, bei 7 war die Medikation gleich geblieben. Von 1331 hausärztlich verordneten Medikamenten wurden 684 (50\%) verändert: 480 Medikamente (36\%) wurden abgesetzt, bei 204 Arzneimitteln (15\%) änderte sich die Dosis bzw. der Hersteller. Neu verordnet wurden 711 Medikamente. Der Anteil von Generika sank von 25,5\% (Praxis) auf 20,2\% in den Krankenhäusern. Von den 284 interviewten Patienten wurden 234 (82\%) über die Medikationsänderungen aufgeklärt. Jeder vierte befragte Patient zeigt sich über die Wirkung des neuen Arzneimittels und den Änderungsgrund informiert. Vollständig über Medikamentenname, Wirkung, Dosis, Einnahmevorschrift und Änderungsgrund informiert war jeder zehnte Patient.

Schlußfolgerung: Die erhebliche Änderung hausärztlich verordneter Medikation im Krankenhaus stellt Hausarzt und Patienten vor Probleme, die nur gelöst werden können, wenn Allgemeinärzte und Klinikärzte bemüht sind, die Zusammenarbeit zu intensivieren und den Patienten in den Verordnungsprozeß einzubeziehen. 
8. Anhang

8.1 Patientendaten

8.2 Medikationsvergleich

8.3 Patientenfragebogen 
MEDIKATIONSVERGLEICH (INN= internationaler Freiname)

\begin{tabular}{|c|c|c|c|c|c|c|c|c|c|c|c|}
\hline $\begin{array}{l}\text { PRAXIS } \\
\text { INN }\end{array}$ & $\begin{array}{l}\text { IKAMENT } \\
\text { Handelsname }\end{array}$ & ATC- Code & Dosis & $\begin{array}{c}\text { Medikament } \\
\text { abgesetzt }\end{array}$ & $\begin{array}{c}\text { Medikament } \\
\text { übernommen } \\
\text { Dosis } \\
\text { unverändert }\end{array}$ & $\begin{array}{c}\text { Medikament } \\
\text { übernommen } \\
\text { Dosis } \\
\text { geändert }\end{array}$ & $\begin{array}{c}\text { gleiche Sub- } \\
\text { stanz } \\
\text { anderer } \\
\text { Hersteller } \\
\text { (Handelsname) }\end{array}$ & INN & $\begin{array}{c}\text { neues } \\
\text { Medikamen } \\
\text { Handelsname }\end{array}$ & ATC-Code & Dosis \\
\hline \multicolumn{12}{|l|}{1.} \\
\hline \multicolumn{12}{|l|}{2.} \\
\hline \multicolumn{12}{|l|}{3.} \\
\hline \multicolumn{12}{|l|}{4.} \\
\hline \multicolumn{12}{|l|}{5.} \\
\hline 6. & & & & & & & & & & & \\
\hline & & & & & & & & & & & \\
\hline
\end{tabular}




\section{Patienten-Daten}

\section{Krankenhaus :}

\begin{tabular}{|l|c|c|c|c|c|c|c|}
\hline $\begin{array}{c}\text { Lfd. } \\
\text { Nr. }\end{array}$ & $\begin{array}{c}\text { Initia- } \\
\text { len }\end{array}$ & $\begin{array}{c}\text { Ge- } \\
\text { schlecht } \\
\mathrm{M}=+ \\
\mathrm{W}=-\end{array}$ & Alter & Sozialstatus & $\begin{array}{c}\text { Vorerkrankungen } \\
\text { (laut KH- Anamnese) }\end{array}$ & $\begin{array}{c}\text { Einweisungs- } \\
\text { Diagnose/n }\end{array}$ & $\begin{array}{c}\text { Klinik- } \\
\text { Diagnose/n }\end{array}$ \\
\hline \\
\hline
\end{tabular}




\section{Patienten- Fragebogen}

Sehr geehrte Patientin, Sehr geehrter Patient !

Dieser Fragebogen wurde im Rahmen einer wissenschaftlichen Studie von der Abteilung Allgemeinmedizin der Universität Göttingen erarbeitet.

In dieser Studie soll geklärt werden, ob und wie Sie im Krankenhaus über Veränderungen bezüglich Ihrer Medikamente unterrichtet wurden. Um diese Daten zu erhalten, sind wir auf Ihre freundliche Mitarbeit angewiesen.

Ihre Angaben werden selbstverständlich anonym und vertraulich behandelt.

Herzlichen Dank für Ihre Hilfe !

1. Sind Sie während Ihres Krankenhausaufenthaltes über Medikamentenänderungen aufgeklärt worden?

\begin{tabular}{|c|c|c|}
\hline & & $\begin{array}{l}\text { O Ja } \\
\text { O Nein }\end{array}$ \\
\hline Wenn Ja, durch wen? & $\begin{array}{l}\text { O Arzt } \\
\text { O Pflegepersonal } \\
\text { O Angehörige } \\
\text { O weiß ich nicht } \\
\text { O Sonstige }\end{array}$ & \\
\hline
\end{tabular}

2. War es für Sie wichtig, aufgeklärt zu werden ?

$\mathrm{O} \mathrm{Ja}$

O Nein

3. Ist es für Sie wichtig, durch den Arzt aufgeklärt zu werden?

O Ja O Nein 
4. Wo erfolgte die Aufklärung ?

O Krankenzimmer

O Arztzimmer

$O$ weiß ich nicht

O Sonstige

5. Wie erfolgte die Aufklärung ?

O bei der Visite, durch den Arzt

$O$ bei der Visite, durch mein Nachfragen

$O$ bei der Besuchszeit, über Angehörige

$O$ weiß ich nicht

O Sonstiges

6. Was wurde Ihnen durch den Arzt mitgeteilt ?

O Medikamentenname

O Wirkungsweise

O unerwünschte Wirkungen

O Dosierung

O Einnahmevorschrift

O Verschreibungs- bzw. Änderungsgrund

O Sonstiges

7. Was ist Ihnen über die neuen Medikamente bekannt?

\begin{tabular}{|ccl|}
\hline Ja & Nein & \\
$\mathrm{O}$ & $\mathrm{O}$ & Handelsname d. M. \\
$\mathrm{O}$ & $\mathrm{O}$ & Dosierung \\
$\mathrm{O}$ & $\mathrm{O}$ & Einnahmevorschrift \\
$\mathrm{O}$ & $\mathrm{O}$ & Verschreibungsgrund \\
\hline
\end{tabular}

8. Nehmen Sie zur Zeit noch zusätzlich vom Hausarzt verschriebene Medikamente ein? 
Wenn Ja, welche?

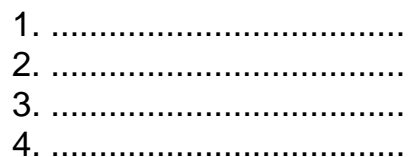

9. Ist das dem Krankenhausarzt bekannt?

$$
\begin{aligned}
& \text { O Ja } \\
& \text { O Nein } \\
& \text { O weiß ich nicht }
\end{aligned}
$$

10. Nehmen Sie rezeptfreie (selbst gekaufte) Medikamente ein ?

$$
\text { O Ja }
$$

$\mathrm{O}$ weiß ich nicht

Wenn Ja, welche?

$$
\begin{aligned}
& 1 . \\
& 2 . \\
& 3 . \\
& 4 .
\end{aligned}
$$

11. Ist das dem Krankenhausarzt bekannt?

$$
\begin{aligned}
& \text { O Ja } \\
& \text { O Nein } \\
& \text { O weiß ich nicht }
\end{aligned}
$$




\section{Literaturverzeichnis}

Barry CA, Bradley CP, Britten N, Stevenson, FA, Barber N (2000): Patients' unvoiced agendas in general practice consultations: qualitative study. BMJ $\underline{320}, 1246-1250$

Bateman DN, Eccles M, Campbell M (1996): Setting standards of prescribing performance in primary care: use of a consensus group of general practitioners and application of standards to practices in the north of England.

BMJ $\underline{46}, 20-25$

Beers MH, Dang J, Hasegawa J, Tamai IY (1989): Influence of hospitalization on drug therapy in the elderly.

J Am Geriatr Soc, $\underline{37}, 679-683$

Beilby J, Silagy C (1997): Trials of providing costing information to general practitioners: a systematic review.

Med J Aust 167, 89-92

Bernatzky G, Pipam W, Pinter G, Mitterschiffthaler G, Likar R (1999): Opioidtherapie bei Ärzten für Allgemeinmedizin in Österreich Ergebnisse und Analyse einer Umfrage.

Schmerz 4 , 266-272

Bijl D, Van Sonderen E, Haaijer-Ruskamp FM (1998): Prescription changes and drug costs at the Interface between primary and specialist care.

Eur J Clin Pharmacol 또, 333-336

Bonetti PO, Hartmann K, Kuhn M, Reinhart WH, Wieland T (2000): Potential drug interactions and number of prescription drugs with special instructions at hospital discharge.

Schweiz Rundsch Med Prax 27, 182-9 
Britten N, Ukoumunne O (1997): The influence of patients' hopes of receiving a prescription on doctors' perceptions and the decision to prescribe: a questionnaire survey.

BMJ $\underline{315}, 1506-1510$

Bundesfachverband der Arzneimittel-Hersteller e. V. Der Selbstmedikationsmarkt in der Bundesrepublik Deutschland in Zahlen 1998: Bundesfachverband der Arzneimittel-Hersteller e. V. , Bonn 1999

Callol JA (1999): Induced prescription in primary health care.

Eur J Gen Pract $\underline{5}$, 49-5

Cantrill JA, Sibbald B, Buetow S (1998): Indicators of the appropriateness of longterm prescribing in general practice in the United Kingdom: consensus development, face and content validity, feasibility, and reliability.

Qual Health Care $\underline{7}, 130-5$

Charles C, Gafni A, Whelan T (1997): Shared decision-making in the medical encounter: what does it mean?

Social Science \& Medicine $\underline{44}, 681-692$

Claoué C, Elkington AR (1986): Informing the hospital of patients' drug regimens. BMJ, 292. $99-102$

Cochrane RA, Mandel AR, Ledger-Scott M, Walker R (1992):Changes in drug treatment after discharge from hospital in geriatric patients.

BMJ $\underline{305}$, 694-696

Coste J, Venot A (1999): An epidemiologic approach to drug prescribing quality assessment: a study in primary care practice in France.

Med Care 37, 1294-307 
Dowell J, Hudson H (1997): A qualitative study of medication-taking behaviour in primary care.

Fam Pract 14, 369-75

Dowell JS, Snadden D, Dunbar JA (1995): Changing to generic formulary : how one fundholding practice reduced prescribing costs.

BMJ $\underline{310}, 505-508$

Dybwad TB, Kjolsrod L, Eskerud J, Laerum E (1997): Why are some doctors highprescribers of benzodiazepines and minor opiates? A qualitative study of GPs in Norway.

Fam Pract $\underline{14}, 361-368$

Fillit HM, Futtermann R, Orland BI, Chim T, Susnow L, Picariello GP, Scheye EC, Spoeri RK, Roglieri JL, Warburton SW (1999): Polypharmacy management in Medicare managed care: changes in prescribing by primary care physicians resulting from a programm promoting medication reviews.

Am J Manag Care $\underline{5}, 587-94$

Frazier LM, Brown T, Divine GW, Fleming GR, Philips NM, Siegal WC, Khayrallah MA (1991): Can physician education lower the cost of prescription drugs?

Ann Intern Med 115. 116-121

Göpel H: Wirtschaftliche Arzneimitteltherapie in der ärztlichen Praxis.

In: Grundlagen der Arzneimitteltherapie, Hrsg.: Dölle W, Müller-Oerlinghausen B, Schwabe U, Kapitel 4.18, Bibliographisches Institut Mannheim,

Zürich 1986, 492-501

Gosney M, Tallis R (1984): Prescription of contraindicated and interacting drugs in elderly patients admitted to hospital.

Lancet $\underline{1984}$, II, 564-567 
Green JA, Chawla AK, Fong PA (1985): Evaluating a restrictive formulary system by assessing nonformulary-drug requests.

Am J Hosp Pharm 느, 1537-1541

Haiko JP, Linden K, Kvist M (1995): Outcomes of referrals from general practice. Scand J Prim Health Care $\underline{13}$, 287-93

Harder S, Thürmann P, Huber Th, Rietbrock N (1991): Prescription of drugs not listet in a clinic`s pharmacopoeia: supervision by clinical pharmacologists.

Eur J Clin Pharmacol $\underline{40}$, 561-564

Hasenbring M, Ahrens S (1986): Zur Arzt-Patient-Beziehung in der ambulanten medizinischen Versorgung.

Psychother Psychosom Med Psychol 트, 274-283

Haynes RB: Einleitung. In: Compliance Handbuch, hrsg.: Haynes RB, Taylor DW, Sackett DL, Oldenbourg Verlag, München, Wien 1982, 12

Himmel W, Kochen MM (1998): Kontinuität und Diskontinuität der hausärztlichen Medikation bei Krankenhauseinweisung.

Z Allgemeinmed $\underline{74}$, 245-250

Himmel W, Lönker B, Kochen MM (1996a): Die Bedeutung der hausärztlichen Verordnung für die stationäre Pharmakotherapie.

Dtsch Med Wochenschr 121, 1451-1556

Himmel W, Tabache M, Kochen MM (1996b): What happens to long-term medication when general practice patients are referred to hospital?

Eur J Clin Pharmacol $\underline{50}, 253-257$ 
Himmel W, Kron M, Thies-Zajonc S, Kochen MM (1997): Changes in drug prescribing under the Public Health Reform Law - a survey of general practitioners" attitudes in East and West Germany. Intern J Clin Pharmacol Ther $\underline{35}$, 164- 168

Hoopmann M, Schwartz FW, Weber J (1995): Effects of the German 1993 health reform law upon primary care practitoners' individual performance: results from an empirical study in sentinel practices.

J Epidemiol Community Health $\underline{49}$, 33-6

Jones MI, Greenfield SM, Stevenson FA, Nayak A, Bradley CP (2001): General practitioners and hospital-initiated prescribing

Eur J Gen Pract 7, 18-22

Jones R, Rawlins MD (1992): Prescribing at the interface between hospitals and general practitioners.

BMJ $\underline{304}, 4-5$

Kochen MM: Umgang mit Arzneimitteln. In: Allgemein- und Familienmedizin, hrsg. Kochen MM, (2. Auflage),

Hippokrates Verlag, Stuttgart 1998

Kruse W, Eggert-Kruse W, Müller S, Kreißler-Haag D, Schlierf G (1990): Vergleich der Aufnahme- und Entlassungsmedikation bei 207 Patienten einer geriatrischen Klinik.

Klin Wochenschr $\underline{68}$, Suppl. XIX, 50-51

Kruse W, Rampmaier J, Frauenrath-Volkers C, Volkert D, Wankmuller I, Micol W, Oster P, Schlierf G (1991): Drug-prescribing patterns in old age. A study of the impact of hospitalization on drug prescriptions and follow-up survey in patients 75 years and older.

Eur J Clin Pharmacol 411, 441-7 
Lucena Gonzalez MI, Ruiz Ruiz J, Andrade Bellido RJ, Hidalgo Sanchez R, Gonzalez Correa JA, Garcia Ruiz A, Sanchez de la Cuesta F (1995): The impact of hospitalization on drug prescription.

Med Clin (Barc) 104, 211-5

Lüttje D, Wiesehahn I (1997): Pharmakotherapie im Alter: Besonderheiten der medikamentösen Therapie bei älteren und hochbetagten Patienten.

Z Allgemeinmed $\underline{73}, 334-342$

Mahdy HA, Seymour DG (1990): How much can elderly patients tell us about their medications?

Postgrad Med J $\underline{66}, 116-121$

Marshall MN (1999): How well do GPs and hospital consultants work together? A survey of the professional relationship.

Fam Pract $\underline{16}$, 33-38

McGavock H, Wilson-Davis K, Rafferty T (1994): A 'compass' for general practitioner prescribers.

Health Trends $\underline{26}, 28-30$

McLeod PJ, Huang AR, Tamblyn RM, Gayton DC (1997): Defining inappropriate practices in prescribing for elderly people: a national consensus panel.

Can Med Assoc J 156, 385-391

Nolan L, O'Malley K (1988): Prescribing for the elderly.

J Am Geriatr Soc 36. 142-149

O'Hanrahan M, O'Malley K (1981): Compliance with drug treatment.

BMJ 283, 298- 300 
Petersen KU (1999): Orginale und Nachahmer.

Med Klin $\underline{95}$, 26-30

Preston C, Cheater F, Baker R, Hearnshaw H (1999): Left in limbo: patients‘ views on care across the primary / secondary interface.

Qual Health Care $\underline{8}, 16-21$

Rokstad K, Straand J, Fugelli P (1997): General practitioners' drug prescribing practice and diagnoses for prescribing: the More \& Romsdal Prescription Study. J Clin Epidemiol $\underline{50}, 485-494$

Rote Liste 1999: Präparate-Statistik, Editio Cantor Verlag, Aulendorf/Württ. 1999, 12

Schmeling-Kludas C, Koch U, Hagenbruch F (1989): Die psychosoziale Lage internistischer Patienten nach der Aufnahme im Krankenhaus-eine empirische Untersuchung.

Psychother Psychsom Med Psychol 39, 75-83

Schmeling-Kludas C, Koch U, Hagenbruch F, Teichert M (1991): Psychosoziale Belastung nach der Krankenhausaufnahme-Ein Vergleich internistischer und chirurgischer Patienten.

Psychother Psychsom Med Psychol 41, 166-175

Schröder H, Selke GW : Arzneimittelverordnungen nach Arztgruppen 1999. Manuskript, Wissenschaftliches Institut der AOK, Bonn 2000

Shabbir MH, Alibhai MD, Ra K, Gary N (1999): Medication education of acutely hospitalized older patients.

J Gen Intern Med 14, 610-616 
Shulkin DJ, Giardino AP, Freenock TFJr., Henriksen DS, Richman C, Friedlander MS, Pandelidis SM, Heywood TJ (1992): Generic versus brand name drug prescribing by resident physicians in Pennsylvania.

Am J Hosp Pharm 노, 625-626

Spagnoli A, Ostino G, Borga AD, Ambrosio RD, Maggiorotti P, Todisco E, Prattichizzo W, Pia L, Comelli M (1989): Drug compliance and unreported drugs in the elderly.

J Am Geriatr Soc 37, 619-624

Stevenson FA, Barry CA, Britten N, Barber N, Bradley CP (2000): Doctor-patient communication about drugs: the evidence for shared decision making.

Soc Sci Med $\underline{50}$ (6), 829-40

Strobach D, Vetter-Kerkhoff C, Bogner J, Breugst W, Schlöndorff D (2000): Patient Medication Counseling - Patientenberatung zur Entlassungsmedikation. Med Klin $\underline{95}$ 548-51

Surendrakumar D, Dunn M, Roberts CJC (1992): Hospital admission and the start of benzodiazepine use.

BMJ $\underline{304}, 881$

Tamblyn RM, McLeod PJ, Abrahamowicz M (1994): Questionable prescribing for elderly patients in Quebec.

Can Med Assoc J 150, 1801-1809

Tomson Y, Wessling A, Tomson G (1994): General practitioners for rational use of drugs.

Eur J Clin Pharmacol $\underline{47}$, 213-219 
Torrible SJ, Hogan DB (1997): Medication use and rural seniors. Who really knows what they are taking?

Can Fam Physician $\underline{43}$, 893-8

Van Hessen PAW, Petri H, Urguhart J (1990): Do prescribed drugs always follow the patients to hospital?

Pharm Weekbl Sci 12, 66-70

Vollmann J, Helmchen H (1997): Aufklärung und Einwilligung (Informed Consent) in der klinischen Praxis.

Dtsch Med Wochenschr 122, 870-873

Webb S, Lloyd M (1994): Prescribing and referral in general practice: a study of patients' expectations and doctors' actions.

Br J Gen Pract $\underline{44}$, 165-9 